\title{
DEFLECTING SMALL ASTEROIDS USING LASER ABLATION: DEEP SPACE NAVIGATION AND ASTEROID ORBIT CONTROL FOR LIGHTTOUCH ${ }^{2}$ MISSION
}

\author{
Massimo Vetrisano ${ }^{1}$ \\ University of Strathclyde, Glasgow, United Kingdom \\ João Branco ${ }^{2}$ \\ GMV SKY-P, Lisbon, Portugal \\ and \\ Daniel Garcia Yarnoz ${ }^{3}$, Joau-Pau Sanchez Cuartielles ${ }^{4}$, Massimiliano Vasile ${ }^{5}$ \\ University of Strathclyde, Glasgow, United Kingdom
}

\begin{abstract}
This paper presents a low-cost, low mass, mission design to successfully intercept and deflect a small and faint, $4 \mathrm{~m}$ in diameter asteroid. Intended to be launched after 2025, the laser-ablating mission, LightTouch ${ }^{2}$ will be used to deflect the orbit of the asteroid by at least $1 \mathrm{~m} / \mathrm{s}$. This will be achieved with a total mission lifetime of less than three years. Analysis includes the initial approach of the spacecraft, the operations of the laser at an optimal spacecraft-to-asteroid distance of $50 \mathrm{~m}$ and the relative orbit of the spacecraft that flies in formation with the asteroid. Analysis includes line-of-sight measurements with radiometric tracking from ground station to improve the trajectory estimate and observability of the spacecraft, collision avoidance and mapping strategies. The spacecraft will also need optimal discrete control. This is achieved by impulse-bit manoeuvres used to account for the perturbations caused by the resultant thrust on the asteroid, plume impingement, laser recoil and solar radiation pressure. The spacecraft controls its trajectory within a 1 m box from the reference trajectory to enable the laser to optimally focussing the laser beam. The proposed approach uses an unscented Kalman filter to estimate the relative spacecraftasteroid position, velocity and perturbative acceleration.
\end{abstract}

\section{Introduction}

$\mathrm{N}_{\mathrm{p}}$ ear Earth Objects (NEO), the majority of which are asteroids, are defined as any minor celestial object with a perihelion less than $1.3 \mathrm{AU}$ and an aphelion greater than $0.983 \mathrm{AU}$. A subclass of these, deemed potentially hazardous asteroids (PHA), are defined as those with a Minimum Orbital Intersection Distance (MOID) from the Earth's orbit less than or equal to $0.05 \mathrm{AU}$ and a diameter larger than $150 \mathrm{~m}$. As of 21st of December 2012, 9432 NEOs have been detected; of those, more than 2600 have a diameter between 0.3 and $1 \mathrm{~km}$, and 1332 are listed as $\mathrm{PHA}^{1}$. Impacts from asteroids of about $1 \mathrm{~km}$ or more in diameter are considered to be capable of causing global

${ }^{1}$ PhD Researcher, Advanced Space Concepts Laboratory, Department of Mechanical \& Aerospace Engineering, University of Strathclyde. massimo.vetrisano@ strath.ac.uk., AIAA Student Member.

${ }^{2}$ Project Engineer, GNC Section, GMV, SKY-P, jbranco@gmv.com.

${ }^{3} \mathrm{PhD}$ Researcher, Advanced Space Concepts Laboratory, Department of Mechanical \& Aerospace Engineering, University of Strathclyde. daniel.garcia-yarnoz@strath.ac.uk.

${ }^{4}$ Research Fellow, Advanced Space Concepts Laboratory, Department of Mechanical \& Aerospace Engineering, University of Strathclyde.jpau.sanchez@strath.ac.uk.

${ }^{5}$ Professor of Space Systems Engineering, Advanced Space Concepts Laboratory, Department of Mechanical \& Aerospace Engineering, University of Strathclyde. massimiliano.vasile@ strath.ac.uk.

1

American Institute of Aeronautics and Astronautics 
climate change and the destruction of ozone, with a land destruction area equivalent to a large state or country. Those with an average diameter of $100 \mathrm{~m}$ can cause significant tsunamis and/or the land destruction of a large city. It is estimated that there are between 30000-300000 NEOs with diameters around $100 \mathrm{~m}$, meaning a large number of NEOs are still undetected.

There is wide interest in the asteroid risks mitigation. Different deflection techniques can be divided into two genres ${ }^{2}$ : contact and contactless. Both the methods are valid, but contact deflection techniques, in the case of impactor, are not effective for every kind of asteroid, such the rubble-pile ones. Moreover this solutions require an object (either a spacecraft/ man-made impactor or another asteroid) to impact the asteroid with high relative velocity. In the likely event the asteroid trajectory is not precisely known, approaching the asteroid fast will reduce the possibility to impact and impart the desired momentum. Other contact techniques which foresee to capture the asteroid and drag it on safer trajectories are difficult to scale-up to larger asteroid missions. Contactless systems are considered in general to be more flexible from this point-of-view; the approach to the asteroid is slower and the deflective action precisely controllable.

In 2012 the European Space Agency (ESA) addressed a technology reference study concerning space mission concepts. The call intended to enable the modification of the orbital dynamics of a 130 tonnes meter-sized asteroids via a suitable contactless deflection technique. The mission is meant to be launch after 2025 , and to have a maximum lifetime of 3 years to impart the asteroid with an overall deviation of $1 \mathrm{~m} / \mathrm{s}$. LightTouch ${ }^{2}$ mission, a consortium, led by University of Strathclyde and formed with partners from EADS Astrium Stevenage, GMV Portugal and the University of Southampton, has been selected to prove the laser ablation proof-of-concept and the feasibility of its in-space demonstration. Laser ablation consists of irradiating asteroid's surface with a laser beam. The absorbed energy increases the temperature of the spot, thus the rock is brought to sublimate, transforming directly from a solid to a gas. The ablated material then expands to form a plume of ejecta which converts the thermal energy into momentum, pushing the asteroid away from its original trajectory.

In order to accomplish such a mission, the spacecraft first needs to detect and approach the asteroid. Once it has completed the asteroid acquisition, the spacecraft can start operating the laser and perform the ablation process. All these different phases are intrinsically affected by uncertainties. The trajectory of the target asteroid, the $4 \mathrm{~m}$ diameter 2006RH120, is uncertain by $5000 \mathrm{~km}(3 \sigma)$ in position at the time of the mission. Moreover the navigation in close proximity of asteroids can be complicated due to the fact that the environment and the response to the ablation are relatively unknown and the dynamics is highly non-linear.

Part of the GNC analysis follows the guidelines for a GNC design for small NEO missions identified by GilFernandez et al. ${ }^{3}$. Typically design and analysis is divided into a Far Approach phase with poor Line-of-Sight (LOS)-only observability and large, usually ground-commanded manoeuvres, a Close Approach phase with the asteroid resolved in the Fields of View (FOV) of the Visual-Based Navigation Sensor (VBS), and either a descent or orbital operational phase.

The ruling dynamics are typically those of the 3-body problem, with the rotation and irregular shape of the asteroid providing an additional challenge because of the changing gravity gradient/forces. The Solar Radiation Pressure (SRP) is also a major source of disturbance and often the GNC analysis can aim for the stabilization around a stable terminator orbit.

In the LightTouch ${ }^{2}$ concept, however, some critical points have very important differences with respect to the typical NEO-encounter mission, while at the same time putting it closer to the challenges faced in Rendez-Vous / Formation Flying missions to non-collaborative targets (for example, orbital debris). Examples of GNC designs for relative motion are ATV, Prisma ${ }^{4}$ and Proba- $3^{5}$ where the Relative Motion Formation Flying and Rendez-Vous GNC, particularly the VBS-only relative navigation experiments, developed in GMV, are extremely relevant applicable to LightTouch2; and Mars Sample Return studies ${ }^{6}$.

The main differences for LightTouch ${ }^{2}$ from typical NEO missions in terms of GNC design are:

- Size of the target Asteroid - Gravity. 2006RH120 is extremely small. Hayabusa was considered a mission to a very small body. Itokawa is a low density rubber-pile asteroid of $535 \times 294 \times 209 \mathrm{~m}$ size. Its gravity force at 10 000 and $50 \mathrm{~m}$ is, respectively, $10 \mu \mathrm{N}$ and $382 \mathrm{mN}$ for the light $400 \mathrm{~kg}$ spacecraft. At the same distances, the 130 Ton-target of LightTouch ${ }^{2}$ will exert a force on the $550-\mathrm{kg}$-spacecraft of only $0.05 \mathrm{nN}$ and $2 \mu \mathrm{N}$, respectively. This is 6 orders magnitude lower than Itokawa. The gravity field of the asteroid, for approach, rendez-vous operations is almost negligible in our case. For what concerns the GNC, it can be considered a small perturbation in the dynamics with respect to the $40 \mu \mathrm{N}$ Solar Radiation Pressure (SRP). The implications in the GNC strategy and design are numerous: during close approach operations, the whole dynamics can be reduced with good approximation from the complex 3-body dynamics to a simpler 2-body dynamics. Linearized dynamic equations can be used in close proximity. A closed form solution to the linearized equations exist and can include SRP ${ }^{7}$. The effect of the rotation of the asteroid in this case is potentially negligible to translational 
relative dynamics if the distance is maintained beyond $50 \mathrm{~m}$ as currently planned so the two problems can be decoupled and the design of the Navigation system can be made more modular. For proximity operation, stable terminator orbits do not exist, therefore, instead of controlling the spacecraft around a stable terminator orbit, the GNC needs to counter-act the effect of SRP to remain in a non-stable operational relative position Safety and control authority also require a different approach to the design. At the proposed operational distance the asteroid barely attracts the spacecraft, although if the spacecraft is placed in between the Sun and the asteroid the SRP is pushing the spacecraft towards the asteroid. Thus, the requirements on the RCS come from the SRP rather than the gravity of the asteroid. During ablation the problem is quite different as the spacecraft is subject to the small but not negligible plume and the asteroid is constantly changing its state of motion. Linearized equations based on the assumption of a constant angular momentum do not consider this added nonlinearity to the motion of the spacecraft that instead need to be considered with care.

- Size of the target Asteroid - Visual Magnitude. The absolute visual magnitude of Hayabusa's Itokawa is 19.2. The 160-m-wide 2002AT4, 21-absolute magnitude target of Don Quijote could be detected from a distance $2500000 \mathrm{~km}$. 2006RH120 worst-case magnitude ( $3 \sigma)$ is 31. The Narrow Angle Camera (NAC) from Rosetta would detect it at $40000 \mathrm{~km}$ from the most favourable illumination angle (sun-asteroid-sc angle of $0 \mathrm{deg}$ ), that is, 3 orders of magnitude below the distance of detectability of what is already considered a small body. Additionally, its ephemeris knowledge are in the same order of magnitude of the distance of detectability. To cover the uncertainty region $(3 \sigma)$ in position of $5000 \mathrm{~km}$ from the detectability distance, the critical detection process (against a bright sky) needs to be repeated for several pointings NAC FOVs, that is, scanning manoeuvres need to be performed, with implications in the early encounter trajectory. At distances of $1 \mathrm{~km}$ the asteroid will be barely resolved in the Wide Angle Camera (WACs) FOV (will span than 10 pixels) and at operational distance it will be 50-pixels wide. This shifts the NAC's relevance as a detection and far approach sensor to serve also approach and proximity. In fact, the $2 \times 4$ asteroid will fit tightly in the FOV of the NAC at proximal distance of $50 \mathrm{~m}$, making the sensor suitable for rotational state estimation and asteroid characterization.

- Duration of Operations. Contrarily to sample return, impact or orbiting characterization missions, LightTouch ${ }^{2}$ aims to remain in an unstable point relative to the asteroid while perturbing its orbit and being effected by perturbing forces and torques from SRP, impingement form the ablation plume, recoil from the laser for 2 years. Even though the forces to be counteracted are small, they are always present. The issue of the life-time of GNC components becomes relevant as the number of RCS actuations rises to the tenths of thousands, the same order of magnitude of their operational limits. The concurrent assessment of activation logic algorithms for position control, similar to those used in attitude control, to save mass, and definition of control windows that are loose enough to allow excursions between actuations, is necessary.

The paper is structured as follows. Section 2 presents an overview of the mission, outlining the mission analysis and the hardware embarked on board. Section 3 describes the GNC strategy which has been envisaged throughout the mission phases. In particular deep space navigation and approach phases are very familiar to those of Formation Flying / Rendez-Vous / Orbital Debris Removal missions, the sub-sections address the points outlined above basing on the expertise from GMV's NEO studies (CHILON, Don Quijote, Marco Polo3) as well as Formation Flying (MSR, Proba-3 4). Section 4 deals with the proximity motion and spacecraft control during the operations. A solution is proposed to estimate the acceleration due to the ablation process, which will give a measure of the momentum transfer onto the asteroid.

\section{Mission Overview}

The target asteroid for the mission is 2006 RH120, a 130 tonnes mass, 4 m diameter body, consistent with the mission requirement from ESA. It was selected mainly due to the relatively good current knowledge of its ephemeris (reported in Table 1). This information would allow reducing the position uncertainty to $5000 \mathrm{~km}(3 \sigma)$ after 2025 , even if no further optical or radar campaign will be available. Moreover it resulted in being a more advantageous target since the optimal transfer opportunity also offers the possibility to see both the asteroid and the spacecraft from Earth during the approach. The baseline transfer considers a launch opportunity in 2027 from a standard PSLV's GTO $\left(200 \times 36000 \mathrm{~km}, 18^{\circ}\right.$ inc). The trajectory was optimized, through patched conics, taking in account the gravitational field of the Sun, solar radiation pressure perturbations, third body perturbations from the Earth and Moon, and gravity losses from non-instantaneous manoeuvres. It resulted in a 296 day transfer, with a departure hyperbolic velocity of $760.2 \mathrm{~m} / \mathrm{s}$. Two apogee raising manoeuvres are performed before escape in order to minimize gravity losses due to non-instantaneous manoeuvres. The overall transfer with respect to the Sun and to the Earth is reported in Figure 1(a),(b). Table 1 shows that a deep space manoeuvre, DSM, is performed 79 days before arrival. 
Table 1. Orbital elements and physical characteristics of 2006 RH120

(http://ssd.jpl.nasa.gov/sbdb.cgi?sstr=2006\%20RH120), and Trajectory Manoeuvres

\begin{tabular}{|c|c|c|c|}
\hline \multicolumn{4}{|c|}{$\begin{array}{l}\text { Orbital Elements at Epoch } 2456200.5 \text { (2012-Sep-30.0) } \\
\text { Reference: JPL } 45 \text { (heliocentric ecliptic J2000) }\end{array}$} \\
\hline Element & Value & Uncertainty (1-sigma) & Units \\
\hline $\mathrm{e}$ & .02447403062284801 & $4.2401 \mathrm{e}-05$ & \\
\hline a & 1.033252056035198 & 1.0251 & $\mathrm{AU}$ \\
\hline$q$ & 1.007964213574672 & 1 & $\mathrm{AU}$ \\
\hline i & .5952660003048117 & $9.4379 e-05$ & $\operatorname{deg}$ \\
\hline node & 51.14334927580387 & $3.8304 \mathrm{e}-05$ & deg \\
\hline peri & 10.14353817485877 & 0.092984 & deg \\
\hline M & 221.2498016727181 & 206.48 & deg \\
\hline$t_{p}$ & $\begin{array}{r}2456348.356001016605 \\
(2013-F e b-24.85600102)\end{array}$ & 1 & JED \\
\hline & 383.6258326667335 & 570.89 & d \\
\hline period & 1.05 & 1.563 & $\mathrm{yr}$ \\
\hline $\mathrm{n}$ & .9384143854377558 & 1.3965 & $\mathrm{deg} / \mathrm{d}$ \\
\hline Q & 1.058539898495724 & 1.0502 & $\mathrm{AU}$ \\
\hline
\end{tabular}

\begin{tabular}{|lcccl|}
\hline & Date & $\begin{array}{c}\text { Days After } \\
\text { Departure }\end{array}$ & $\begin{array}{l}\Delta \mathbf{v} \\
(\mathbf{m} / \mathbf{s})\end{array}$ & $\begin{array}{l}\text { Mass } \\
\text { After }\end{array}$ \\
\hline Departure & $2027 / 11 / 18$ & 0 & 792.4 & $\begin{array}{l}835.0 \\
\mathrm{~kg}\end{array}$ \\
\hline DSM & $2028 / 6 / 22$ & 217 & 186.3 & $\begin{array}{l}787.0 \\
\mathrm{~kg}\end{array}$ \\
\hline $\begin{array}{l}\text { Arrival } \\
\text { (RVM)* }\end{array}$ & $2028 / 9 / 9$ & 293 & 395.2 & $\begin{array}{l}694.1 \\
\mathrm{~kg}\end{array}$ \\
\hline
\end{tabular}

Absolute Magnitude: 29.527; Rotation Frequency 21.8 rev/h

Notice that the rendez-vous manoeuvre (RVM) does not aim the asteroid but at rendez-vous condition, designed to prepare the final approach (see right Figure 1(c)).
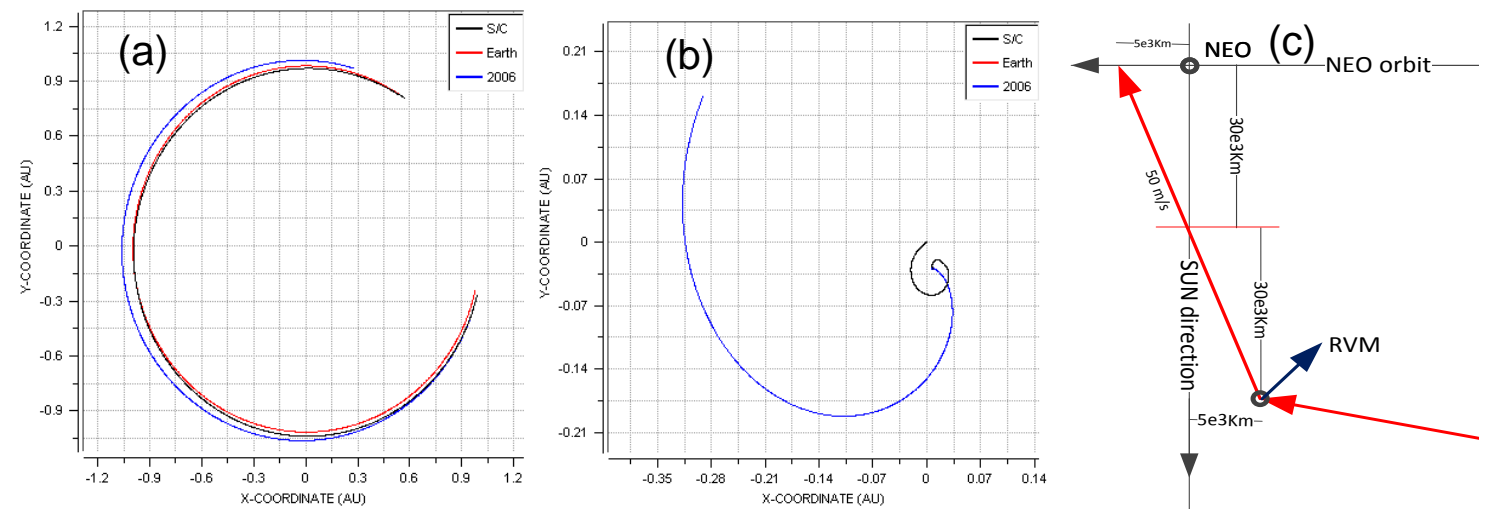

Figure 1. X-Y Projection of Trajectory in Sun-Centred (a) and Earth-Centred (b) in Ecliptic System 2000, and Rendez-Vous Condition (c)

In order to maximize the ablation process and minimize the risk associated to flying in the clouds of ejecta plume closer to the asteroid, the spacecraft will need to fly at a constant range of $50 \mathrm{~m}$ with small variation. The laser system is able to cope with $1 \mathrm{~m}$ oscillation, by adjusting it optics. It is, then, task of the GNC to reduce the number of such corrections (see Section III.E).

Figure 2 shows two different configurations which have been considered during the study: 1) trailing configuration - the spacecraft is flying in formation with the asteroid along track; 2) radial configuration - the spacecraft is in between the asteroid and the Sun along the Sun-asteroid direction (slightly shifted to avoid solar radiation to push the spacecraft onto the asteroid, if no control is maintained). In both cases the spacecraft mass is approximately $600 \mathrm{~kg}$ at arrival with a $7.4 \mathrm{~m}^{2}$ solar arrays. The trailing configuration mitigates the contamination which affects the optics, radiators and solar arrays, while the radial configuration reduces the number of actuations by balancing the forces acting on the spacecraft. 

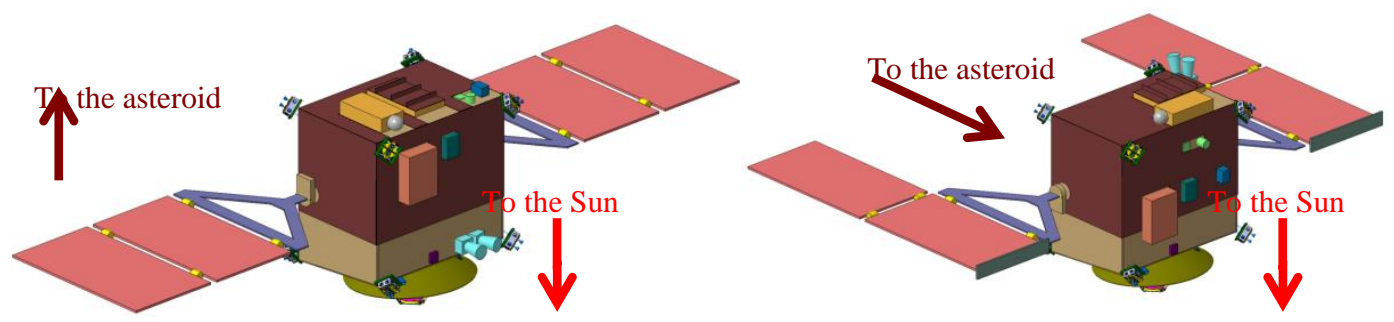

Figure 2: Spacecraft configuration for radial and trailing configuration (courtesy of EADS Astrium)

The GNC Hardware selection for the mission followed traditional guidelines for NEO missions, the specificity of this particular one (close distance to Earth, NEO is the size of the S/C) being that its challenges are similar to those of a rendez-vous with a passive / uncollaborative target (like a rendez-vous with a canister in the MSR mission or space debris removal).

- Star trackers (STR) / Gyroscopes (IRU) assembly for attitude determination and Reaction Wheel assembly for attitude control / stabilization

- RCS thrusters for position control and coarse attitude control/reaction wheel desaturation

- Vision Based Sensor assembly:

-High aperture telescopic Narrow Angle Camera for detection and navigation (LOS-measurement) at far distances, asteroid characterization

- Wide Angle Camera for navigation at close distances / redundancy / safety

- Ranging Sensor, together with LOS measurement from the camera, allow a robust full relative state observibility with the object of unknown brightness/size.

An impact sensor, heritage of the mission Rosetta will be used to measure the momentum created by the ablated ejecta. This is needed to ensure the system's capability of being able to modify the orbit of the asteroid by $1 \mathrm{~m} / \mathrm{s}$ (in three years). The system is used to detect the optical transit and cross-section of each particle entering the instrument, and allows determining the force exerted on the spacecraft from the plume ejecta.

\section{GNC Strategy and Mission Timeline}

With reference to Figure 3, which reports the mission profile with respect to the Sun from launch till the end of life after 3 years, the mission will be conceptually divided in eight phases characterized by different operational modes.

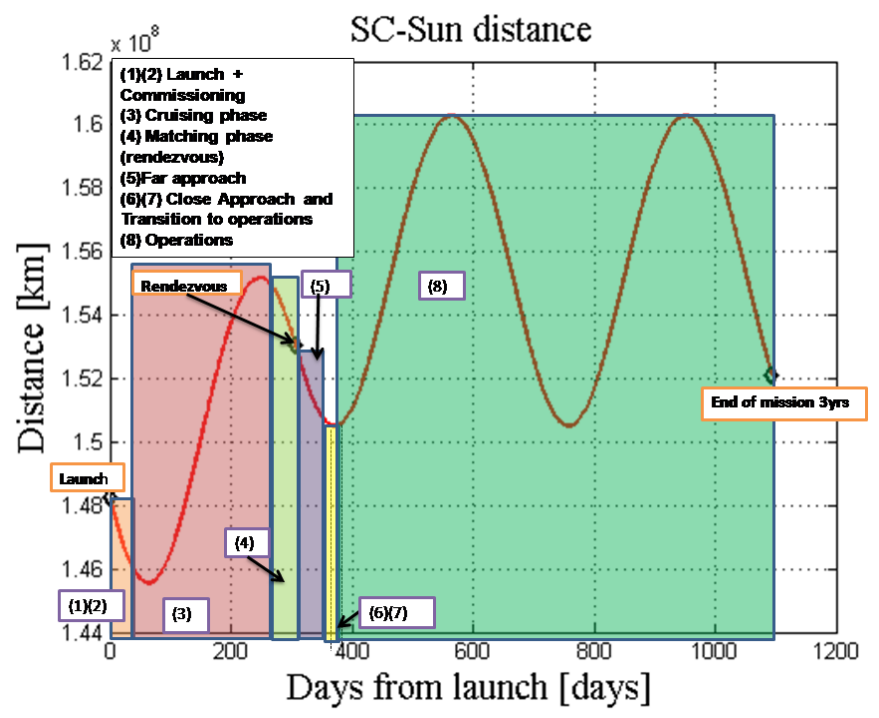

Figure 3: Mission phases with respect to the Sun distance 
1. Launch: Escape is achieved by means of the main chemical engine. Before the spacecraft is injected into the interplanetary transfer trajectory by the hyperbolic escape manoeuvre, 2 apogee raising manoeuvres are performed to raise the apogee from the GTO in which the launcher inserts the spacecraft.

2. Commissioning: Immediately after separation, the spacecraft will autonomously de-tumble, deploy its solar arrays and acquire a coarse three-axis stabilised Sun-pointing attitude. After launch, a tracking campaign will be performed in order to verify the interplanetary transfer trajectory and, if required, implement a correction manoeuvre TCM. Before putting the spacecraft into hibernation mode, all its functions will be checked and the payload will be commissioned.

3. Earth to Asteroid Cruise/Interplanetary: During the cruising phase the spacecraft will be in hibernation mode and no ground support will be required. The spacecraft will be resumed for 2 weeks to allow the spacecraft to perform the single DSM, 79 days before arrival. After the DSM, a tracking campaign determines the spacecraft's orbit. A TCM, 7 days after DSM is possibly performed. The transfer lasts for 296 days, and the spacecraft arrives at the asteroid approximately 90 days before its perihelion.

4. Early Encounter/Arrival phase: One month before the designed Rendez-Vous Manoeuvre, RVM, (considered the arrival time) the spacecraft will be resumed. Ground station will track the spacecraft to implement possible corrections and if necessary re-plan the rendezvous manoeuvre. RVM leaves the spacecraft trajectory in a sunasteroid direction in order to reduce the illumination angle (sun-asteroid-spacecraft), which results in a higher apparent visual magnitude. 3 days before RVM, occasional picture will be taken and relayed to calibrate the NAC, WAC and STR. The main engine will perform the $391 \mathrm{~m} / \mathrm{s}$ manoeuvre at a distance of approximately $60,000 \mathrm{~km}$ from the estimated position of the asteroid, thus reducing the relative velocity with respect to nominal asteroid's trajectory to $50 \mathrm{~m} / \mathrm{s}$. A ground supported campaign will verify the spacecraft's trajectory. Then the spacecraft will enter a target detection mode to acquire and identify the asteroid LOS in the NAC, and the asteroid's ephemeris will be improved. LOS measurements will be acquired from different angles and combined with radiometric navigation data. After 1 week the acquisition of the asteroid is assured. 2 OpticalNav-Based Early-Encounter-TCM, EE-TCM-1 (10 days after RVM), EE-TCM-2 (12 days after RVM) will reduce relative position uncertainty from $5000 \mathrm{~km}$ by three orders of magnitude to $<10 \mathrm{~km}$. The phase concludes with Far Approach Preparation Manoeuvre FAPM, 14 days after RVM at about $5000 \mathrm{~km}$ distance. The Early Encounter strategy is described in detail in Section A.

5. Far-Approach (11 days): The far approach trajectory phase starts with clean-up of FAPM. A sequence of 3 subsequent manoeuvres by the RCS will reduce the relative distance from $5000 \mathrm{~km}$ to $10 \mathrm{~km}$ to allow start of autonomous operation:

- 1 day later, Far Approach Start Manoeuvre, FASM

- 5 days later, Far-Approach-Mid Manoeuvre, FADM, to compensate SRP

- 5 days later, arrival, Far-Approach-End Manoeuvre FAEM

This phase ends at the Approach Transition Point (ATP), 11 days after the beginning of this phase. At this stage (about $10 \mathrm{~km}$ from the asteroid) the NAC is able resolve the target to $>10$ pixels. At ATP the relative trajectory knowledge will accurate to $1 \mathrm{~km}$ in position, thanks to a high number of LOS measurements taken from different angles. The Far Approach strategy is described in detail in Section B.

6. Close-approach (11 days): The close approach trajectory phase starts at ATP. In this phase the spacecraft will autonomously approach the asteroid to ranging sensor acquisition. A sequence of dog-leg manoeuvres through way-points will be performed to allow LOS-based relative navigation accuracy to improve to $<20 \mathrm{~m}$ in the range direction; at the same time the spacecraft will acquire ranging sensor and arrive to Hold Point 1, HP1, at $300 \mathrm{~m}$ from target. Final relative navigation accuracy shall be almost optimal (a part from fine-calibration). The sequence of operations is:

- Preparation of the autonomous LOS-based-GNC.

- Dog-Leg Autonomous Approach Segment, starting 2 days later

- Spacecraft autonomously travels through 6 way points, 1 per day, to reach a $1 \mathrm{~km}$ distance from asteroid (Sun-NEO-SC angle of $45 \mathrm{deg}$ )

- Waypoint 6 coincident with Hold Point 1 HP1 where it holds one day in preparation for next step

- 9 days after start, the Final Close Approach Segment starts, with an autonomous approach to $300 \mathrm{~m}$ from asteroid (Sun-NEO-SC angle of 0 deg ).

- This 6-hour approach is supervised from ground. During the approach, the ranging sensor is acquired

Then, for 2 days, GNC equipment status is verified while autonomous GNC controls the spacecraft to remain within a $5 \mathrm{~m}$-wide control box. The end of the close autonomous approach is 11 days after it started. The Close Approach strategy is described in detail in Section C. 
7. Transition to the Close operative phase (26 days) starts at the end of Final Close Approach Segment. The spacecraft follows subsequent hyperbolic arcs until it is about 25 radii $(50 \mathrm{~m})$ from the asteroid, in the proximity operations point. During this phase, GNC system is tested and calibrated, including algorithms, sensors and actuators, and optimal asteroid orbit determination is performed. Up to the acquisition of the final relative position the phase lasts 26 days.

8. Operative phase starts at the end of the third GNC test. The bulk of the operations towards the main Mission Objective in which the laser efficiently imparts $\Delta \mathrm{v}$ to the asteroid by ablating it. The spacecraft will actively control relative position and attitude and measure the imparted $\Delta \mathrm{v}$.

- Sequence of 90-day ablation plus 10 day-measurement process

- The ablation causes the asteroid to accelerate $\sim 0.05 \mu \mathrm{m} / \mathrm{s} 2$

- Laser operates for 6 full days continuously, then data is relayed to Earth for 1 day (to Harwel), including camera images, health, house-keeping and radiometric navigation data. 13 sequences of ablation/data-relay operations are performed in a batch.

- Followed by a 10-day a radiometric navigation campaign using radiometric and ranging from the Harwel ground station and $2 \triangle \mathrm{DOR}$ measurements from ESA's DSA.

- Three of these sequences complete the baseline mission. Nominally, all mission objectives shall be attained at this point, including the successful change of NEO velocity by $1 \mathrm{~m} / \mathrm{s}$, full precise characterization of the asteroid rotational and orbital state confirmation of the change in orbit, as well as additional scientific objectives.

- This will happen 723 days after launch, at an Earth-NEO distance of 0.404 AU. The Full Operational Phase strategy is described in detail in Section D.

Due to the continuous change of the orbit of the asteroid, and the perturbations acting on the spacecraft (recoil of the laser, solar radiation pressure and plume impingement) the orbit of the spacecraft will need to be adjusted to maintain the close formation motion. The control, implemented by RCS thrusters, aims at maintaining the spacecraft's relative within a $\pm 1 \mathrm{~m}$ control box. The on-board system is able to estimate the accelerations the spacecraft. This information can be integrated in time to measure the overall delta-v imparted to the asteroid by the ablation. A second method to assess this value is through the ground-based radiometric measurements from Earth, where the position of the spacecraft can be tracked to the accuracy required to establish the new ephemerides of the asteroid.

Each of the operational phases of the GNC is described and analysed in detail in the following sub-sections.

\section{A. Early Encounter}

The initial early encounter condition (set by the RVM) and consequent trajectories are selected to:

- Optimize detection of the faint asteroid by the NAC. A detection at maximum detectability range allows to either minimize or exclude a scanning manoeuvre, and allows optical-navigation-based control action for relative trajectory control to be taken earlier, thus minimizing consumption.

- Allow measurements from different directions $(90 \mathrm{deg})$ to be combined with radiometric data to allow combined radiometric / optical navigation and thus concurrently improve the asteroid's ephemeris knowledge and the relative position and velocity knowledge by three orders of magnitude (to $<10 \mathrm{~km} 3 \sigma$ ), to support the start of the Far Approach phase.

- Contribute to minimize time of arrival. There are advantages of starting the ablation as soon as possible, when the NEO is closest to its perihelium and to Earth, for power-efficiency, dynamic efficiency of the push, and communications (it's the closest to Earth at arrival).

- Minimize $\Delta \mathrm{v}$ of RVM and subsequent Far Approach manoeuvres.

The absolute magnitude of the 2006RH120 is 29.52 ( $\sigma$ 1.25). Assuming the worst case $3 \sigma$, the early encounter is sized for a 32 absolute magnitude, very faint asteroid. The sizing case for camera's maximum detection range was to assume it can detect a maximum relative magnitude of 13.5 (slightly conservative with respect to the 14 detectable by the baseline NAC).

The relative visual magnitude of a celestial object depends on its phase angle (optics-target-Sun), distance to Sun, distance from camera to target. Figure 4 shows the area from where a camera of 13.5 can detect an asteroid of a certain absolute magnitude. Highlighted in blue is the area in space from where a target with an absolute magnitude of 31 can be detected. The Early Encounter trajectory, overlapped with the plot, arrives at the phase angle of $0^{\circ}$ at $30000 \mathrm{~km}$, being able, at this point to detect the worst-case-faint asteroid. The whole trajectory lies in the area of detection of the nominal magnitude asteroid. After the entering the worst case 3- $\sigma$ area of the asteroid, it remains within it because as the phase angle becomes closer to $90^{\circ}$, the range decreases. 


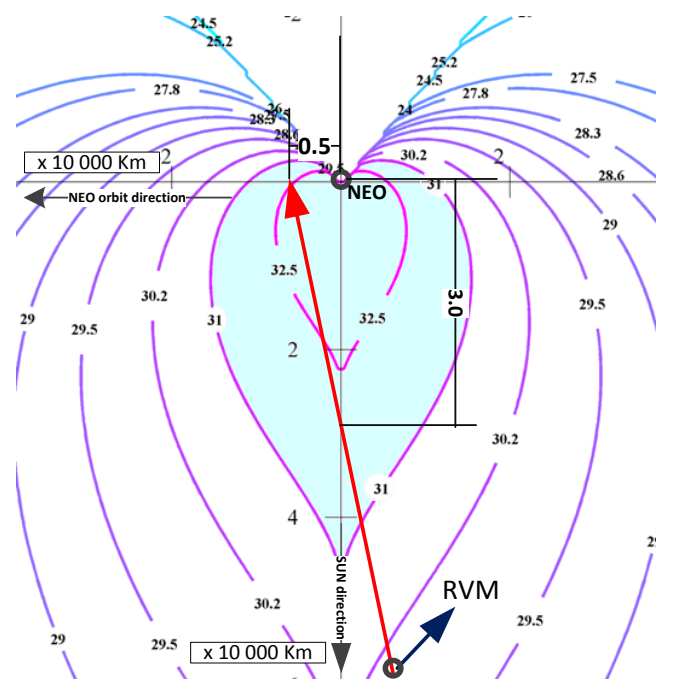

Figure 4. Detectible Area

The area where a spacecraft can approach the asteroid while seeing it lies mostly in the direction of the Sun (opposite direction when compared to the previous plot). The asteroid is in this case is assumed to be fixed and constantly at $1 \mathrm{AU}$.

Detection shall be tried from the start of the Early Encounter phase, and is assured, at most, at its middle point, at $30000 \mathrm{~km}, 0$ degree phase angle to the NEO. From this point onwards TCM can be programmed to take into account the improvement on relative precision due to optical LOS navigation.

In order to minimize or exclude a scanning manoeuvre, detection shall be performed as soon as possible, as illustrated in Figure 5.

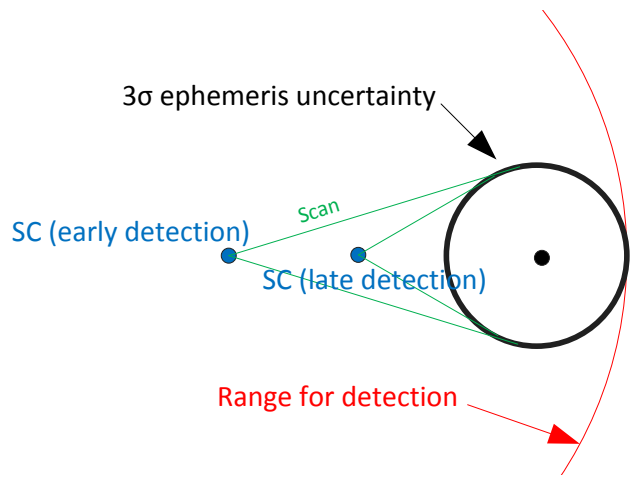

Figure 5. Angular search area depending on detecting distance

At $60000 \mathrm{~km}$ of distance to the asteroid, where RVM is programmed, and where asteroid can be nominally detected, the NAC's FOV of 2.95 deg covers an area of $3000 \mathrm{~km}$. To cover the $3 \sigma$, or $5000 \mathrm{~km}$ of ephemeris uncertainty of the asteroid, a small scanning manoeuvre (4 pictures) would be necessary.

At $30000 \mathrm{~km}$ of distance, where detection of even the faintest $(3 \sigma)$ asteroid is possible, the NAC's FOV covers an area of $1540 \mathrm{~km}$, about the $1 \sigma$ value of ephemeris uncertainty. In the combined worst case for ephemeris uncertainty and faint asteroid, the scanning manoeuvre would have to cover a 9.5 deg FOV (50 pictures would be necessary).

To assure early detection against a starry background, a stacking of images of long exposure times is required. Transversal velocity shall be limited to avoid the asteroid to move one pixel during the exposure. Typical values for long exposure times are 2.5 seconds. The pixel scale is $25 \mu \mathrm{rad}$. At $30000 \mathrm{~km}$, the designed transversal velocity of $8.3 \mathrm{~m} / \mathrm{s}$ causes the target to move only $7 \mu \mathrm{rad}$ in 25 seconds, little enough that assures all the photons from exposure fall in the same pixel. 
This phase is also the most stringent in terms of pointing stability - separation between the instantaneous actual pointing and the short time average pointing direction. A pointing stability better than the point spread function (slightly larger than the pixel size) shall be achieved during one exposure time. Considering 2.5 seconds of exposure, this amounts to a pointing stability requirement of $2 \mathrm{arcsec} / \mathrm{sec}$ (LOS). This requirement one order of magnitude looser than for a typical rendez-vous mission (Proba-3, Xeus).

After all considerations the resulting trajectory is shown in Figure 6(a).

- The Radiometric-based RVM aims at a point in the perpendicular plane to the sun direction, $5000 \mathrm{~km}$ (the initial ephemeris uncertainty) distance from the asteroid in its orbital direction

- The Early Encounter lasts 2 weeks, so this equates to an approach velocity of $50 \mathrm{~m} / \mathrm{s}$. The RVM $\Delta \mathrm{v}$ is of $391.7 \mathrm{~m} / \mathrm{s}$.

- Scanning - batches of long-exposure $(2.5 \mathrm{sec}) 10$ images in one hour are collected every 10 hours.

- After target acquisition has been confirmed (which should occur right after RVM but at most occurs after 7 days), 1 image per hour is collected.

- Combined radiometric and LOS-Navigation processing is performed on ground.

- 2 TCM are programmed (making use of the Optical Navigation) and executed.

- EE-TCM-1 , performed 10 days after RVM. At this point LOS measurements to the asteroid have been obtained from an amplitude of angles of $25 \mathrm{deg}$. (nominally, worst-case , $15 \mathrm{deg}$ ). The nominal distance is $17000 \mathrm{~km}$, and the relative position accuracy has been improved to $<500 \mathrm{~km}$.

- EE-TCM-2 , 12 days after RVM, LOS measurements have been taken from an amplitude of angles of 40 degrees (nominally, worst-case, 30 degrees). The nominal distance is $8600 \mathrm{~km}$ and the relative position accuracy has been improved to $<200 \mathrm{~km} 3 \sigma(4 \%$ of the initial). Illustrative example in

- The Far Approach Preparation Manoeuvre (FAPM), executed with the main engine 14 days after RVM, when the phase angle is 90 degrees, reduces the relative velocity to leave the spacecraft in the same orbit of the asteroid, $5000 \mathrm{~km}$ ahead. The relative position | velocity accuracy shall be better than $10 \mathrm{~km} \mid 10 \mathrm{~mm} / \mathrm{s}$ $(3 \sigma)$ at this point

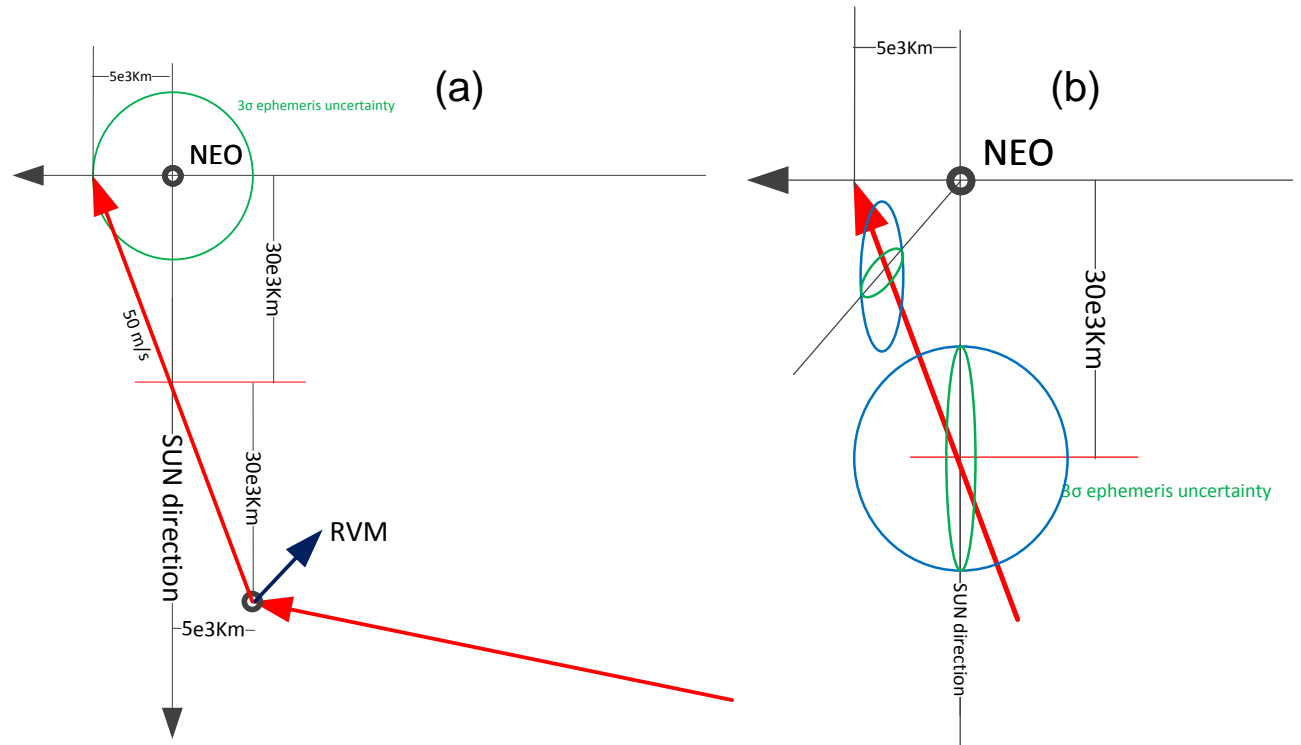

Figure 6. Early Encounter Trajectory (a) and Schematic of the proposed optical navigation: initial knowledge (blue) improves as LOS measurements are processed (green) (b)

\section{B. Far Approach}

The far approach aims at arriving at a relative range and relative accuracy that will allow the start of the autonomous operations of close approach. The objective is again to concurrently lower the range to the asteroid and collect LOS measurements from different angles to improve the relative navigation accuracy to the $\sim 1 \mathrm{~km}, 1 \mathrm{~mm} / \mathrm{s}$ that are necessary for the start of autonomous approach. At start of Far Approach the asteroid still spans less than 1 pixel in the FOV. The differential gravity acceleration of $\sim 1 \mu \mathrm{m} / \mathrm{s}^{2}$ dominates the dynamics earlier until $<500 \mathrm{~km}$ where it becomes lower than the SRP $(\sim 0.07 \mu \mathrm{m} / \mathrm{s} 2)$. 
The design of the far approach trajectory was designed as a dogleg to observe the asteroid from a phase angle from 90 degree to 0 (final). The trajectory is such that the manoeuvres become smaller as the distance decreases.

After one day of clean-up of the FAPM with RCS and preparation, the Far Approach Start Manoeuvre (FASM) is commanded from ground and executed. The manoeuvre takes in account the best estimate of Solar Radiation Pressure and relative motion dynamics. In a first segment the targeted point is in the straight line between Far Approach Start Point (5000 ahead in the trajectory of the asteroid) and the Far Approach End Point (10 km in the Sun-target direction), at $15 \mathrm{~km}$ distance from the latter(see Figure 7). The gravity gradient and SRP cause the resulting trajectory not to be a straight line like shown in the simplified Figure 7 but an arc (see Figure 8) with a significant excursion $(37 \mathrm{~km})$.

The first segment implies a $\Delta \mathrm{v}$ of $11.5 \mathrm{~m} / \mathrm{s}$ executed in 14 seconds with the main engine. The second segment breaks the $11.5 \mathrm{~m} / \mathrm{s}$ with the main engine and (now using the RCS) imparts a $35 \mathrm{~mm} / \mathrm{s}$ approach velocity. The gravity gradient becomes negligible at this point $(<0.001 \mu \mathrm{m} / \mathrm{s} 2)$ but the SRP causes an excursion in the trajectory of $\sim 2 \mathrm{~km}$ with respect to a straight line (Figure 9). The second segment allows covering the remaining 60 degrees of the observation arch in the same 5 days the first segment covered 5 degrees. The second segment's approach velocity is much lower.

For almost all of the first segment of the Far Approach, the asteroid lies within a single pixel of varying brightness. Two NAC images per day are relayed to the ground, allowing not only an optimized relative trajectory computation, but also, concurrently, an initial refinement of the assessment of the absolute magnitude of the asteroid and its variation with respect to distance (which can be useful to determine range). Notice, however, that due to the rotation of the asteroid and the fact that it is observed from different phase angles, this assessment is still coarse.

The relative magnitude of the asteroid is still low enough $(\sim 10)$ to allow detection of stars in the background so, not only it can be calibrated with the star trackers and WAC, but also the attitude / LOS measurements can be negligibly affected by misalignment of the sensors.

At the second segment the asteroid already spans more than 25 pixels in the FOV. At this point, like in the close approach phase, the LOS precision is affected by the offset between CoB and CoM. This effect is more accentuated in the initial days of the second segment, becoming less relevant when the phase angle becomes close to zero.

At the end of the far approach phase, the spacecraft lies in the sun-asteroid direction at $10 \mathrm{~km}$ range. The asteroid spans in an area of at least $8 \times 8$ (64 pixels) in the NAC and is already visible in the WAC. A coarse characterization of its size has been performed and calibration of all the sensors is achieved.

The relative navigation provides an overall accuracy $3 \sigma$ of $1 \mathrm{~km}, 1 \mathrm{~mm} / \mathrm{s}$.

FAR APPROACH

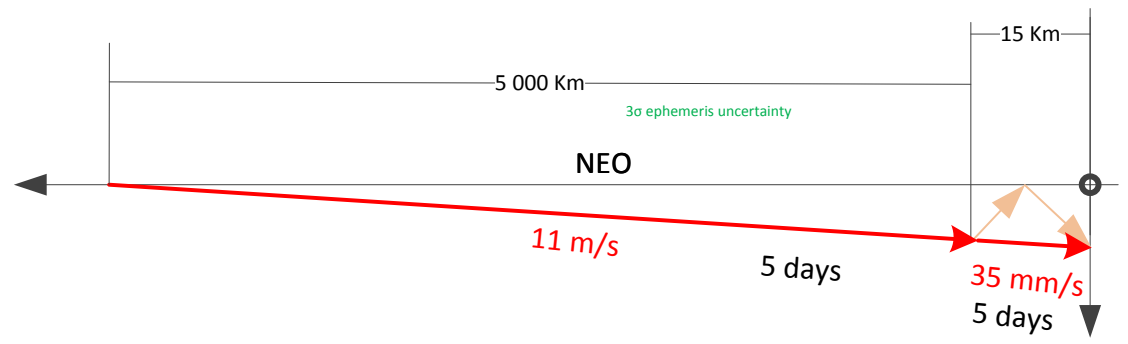

Figure 7. Far Approach Trajectory (simplification)

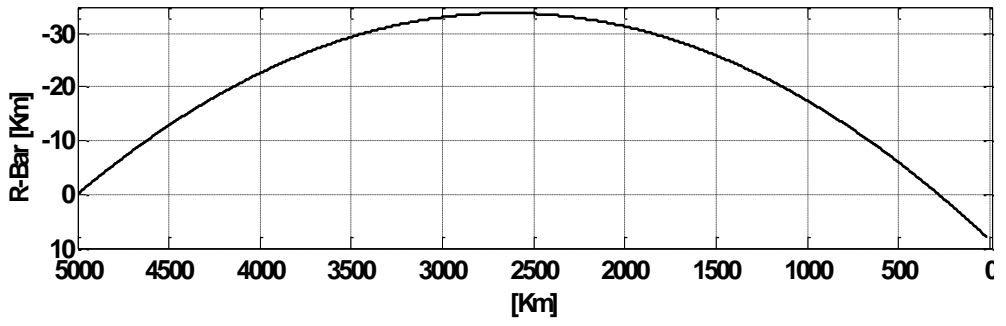

Figure 8. First segment (5 day arc) of Far Approach trajectory, showing the curvature of the trajectory due to SRP and gravity gradient. 


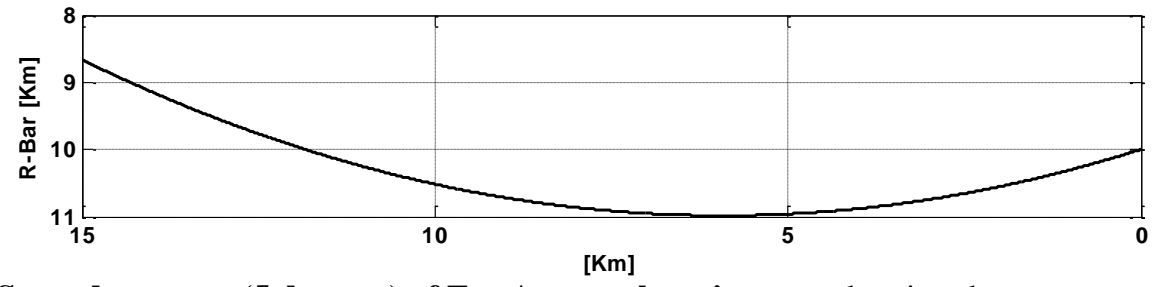

Figure 9. Second segment (5 day arc) of Far Approach trajectory, showing the curvature of the trajectory due to SRP and gravity gradient.

\section{Close Approach}

The close approach main objective is to safely and quickly deliver the spacecraft to within range of the ranging sensor for the proximity operations. The $38 \mu \mathrm{N}$ SRP would cause the spacecraft to move $1 \mathrm{~km}$ and $12 \mathrm{~mm} / \mathrm{s}$ in 2 days towards the asteroid, so it is essential to have autonomy in the GNC for the approach.

The design of an approach profile for LOS navigation presents roughly the same challenges, and again, a series of dog-leg manoeuvres are a robust option. As the range to the asteroid decreases so should the magnitude of the manoeuvres. The smallest considered size for the asteroid is $2 \mathrm{~m}$ of diameter, which will span $8 \times 8$ pixels in the NAC at the $10 \mathrm{~km}$ start and $266 \times 266$ at its $300-\mathrm{m}$ range end.

The autonomous GNC, based on on-board image processing (relying mostly on brightness centroiding), though highly supervised from the ground (which receives 6 NAC, 6 WAC images a day), after a one-day clean-up of FAEM and waiting from go from ground, takes the spacecraft through a 6 waypoints in 6 days to the Hold Point 1 , at $1 \mathrm{~km}$ from the asteroid, where it holds until a command from the ground to perform a 6 hour supervised straightline approach to a $300 \mathrm{~m}$ range in the Sun-NEO direction.

Figure 10 shows the way points and approach velocities for each of the 2-day segments. Differential-corrective guidance (fixed-time-of-arrival) including the model of solar radiation pressure for relative motion is employed to take the spacecraft through the 90 degree amplitude dog-legs. To further assist the observability in the range direction at each way point (including the intermediate WP1, WP3 and WP5), a breaking to zero followed by a new impulsive manoeuvre is performed - a knowledge of $10 \%$ of the value of an transversal impulsive $\Delta \mathrm{v}$, and LOS rate measurements, would, with no other contributions, lead to a knowledge of $10 \%$ in range (e.g., $100 \mathrm{~m}$ at $1 \mathrm{~km}$ ).

A Kalman filter will estimate the state composed of relative position and velocity, and augmented with an acceleration bias, the CoM to $\mathrm{CoB}$ offset, both modelled as exponentially correlated random variables. A linear solution to the relative motion in elliptical orbits under solar radiation pressure - the Yamanaka-Ankersen equations ${ }^{8}$ - can be effectively used. The manoeuvre execution information (from actuation manager and possibly IRU), and manoeuvre execution errors are accounted for. While the major source of perturbation is the Solar Radiation pressure, the most important source of measurement error is the fact that the CoM doesn't correspond to the measured brightness centroid $(\mathrm{CoB})$.

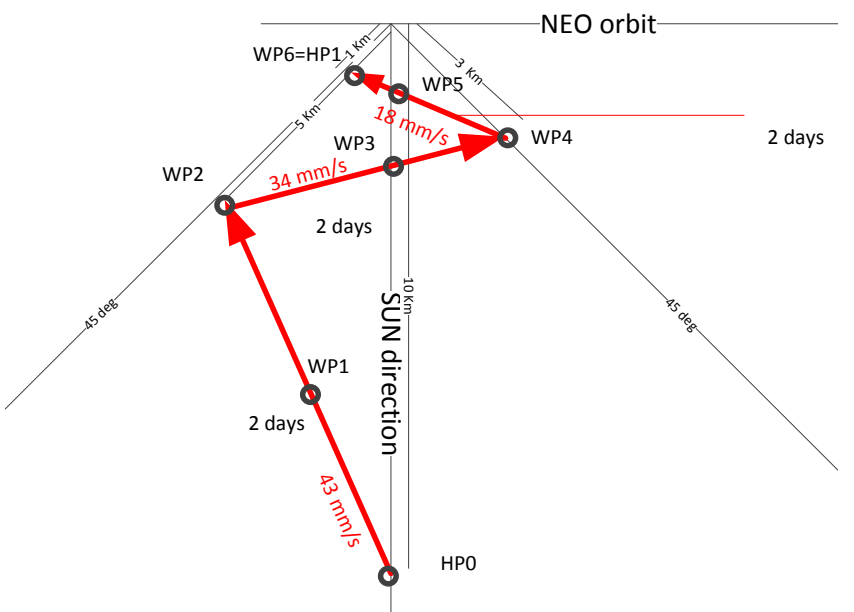

Figure 10. Close Approach way points of Dog-Leg Autonomous Approach Segment

This is particularly relevant in the velocity estimation, as the maximum rotation rate of the asteroid is expected to be $21 \mathrm{rot} /$ hour - if a drift of $0.1 \mathrm{~m}$ in CoB-CoM offset is caused by one rotation, then an unfiltered estimate from 
LOS rate would provide an apparent lateral velocity of $3 \mathrm{~mm} / \mathrm{s}$. This, however, is compensated through a series of methods:

- Inclusion of phase/frequency modelling of the state and processing within the filter

- Pre-processing of LOS / LOS-rate through band-pass / averaging. The adaptive filters can make use of guesses of rotational rate to improve the performance.

- Determination of this offset through feature-point-based asteroid rotational state characterization

Regarding the latter, and especially in the later moments of the close approach, the autonomous Feature Extraction Integrated Circuit is able to provide azimuth/elevation to distinguishable feature points in the asteroid, a number of which $(\sim 10)$ can quickly be processed on-board to characterize the rotation of the asteroid. This characterization is used to feed a Phase-Lock/Frequency-Lock that optimizes the filtering.

Notice as well that the effect of drift of CoB is only significant when in large phase angle (close to WP2,4 and 6). The effect is illustrated in Figure 11.
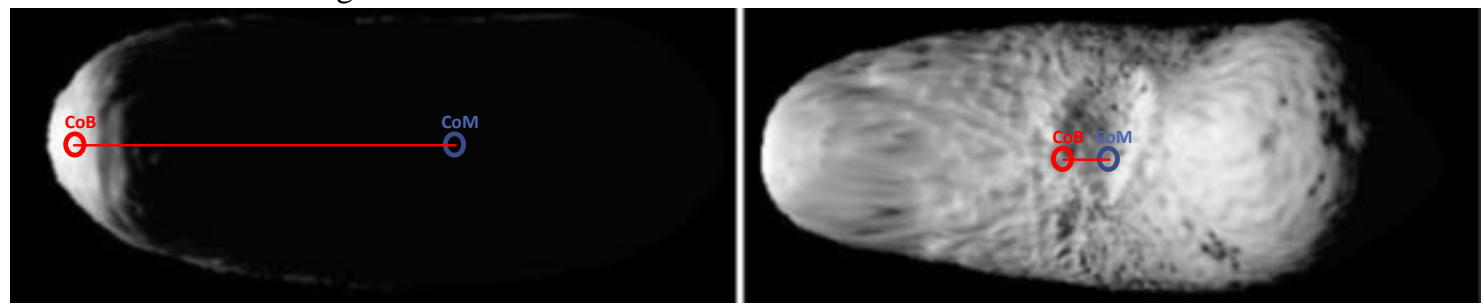

Figure 11. Asteroid observed from $60 \mathrm{deg}$ phase angle, still geometry save for rotational state

As was the case for HP0, the autonomous GNC will hold the spacecraft within a $20 \mathrm{~m}$ wide control box around the HP1 at $1 \mathrm{~km}$ from the asteroid, waiting for command from ground to proceed. The hold guidance law is explained later in the paper when discussing transition to operations phase. At this point, the estimation error in relative velocity shall be below $0.1 \mathrm{~mm} / \mathrm{s}$, while the range direction error is lower than $10 \mathrm{~m}$.

When ground issues the command, an impulsive manoeuvre of $38 \mathrm{~mm} / \mathrm{s}$ puts the spacecraft in a slow trajectory towards the close approach final point, which should be reached within 6 hours of ground-supervised autonomous operation. During this segment of the trajectory, the ranging sensor shall be acquired. At $300 \mathrm{~m}$, the sensor status can be verified and its measurements integrated in the Relative Navigation Filter. Then, during 2 days GNC equipment status is verified while autonomous GNC controls the spacecraft to remain within a $5 \mathrm{~m}$-wide control box. The end of autonomous approach takes place 11 days after it started. The knowledge accuracy shall be $<3 \mathrm{~m},<$ $0.3 \mathrm{~mm} / \mathrm{s}(3 \sigma)$.

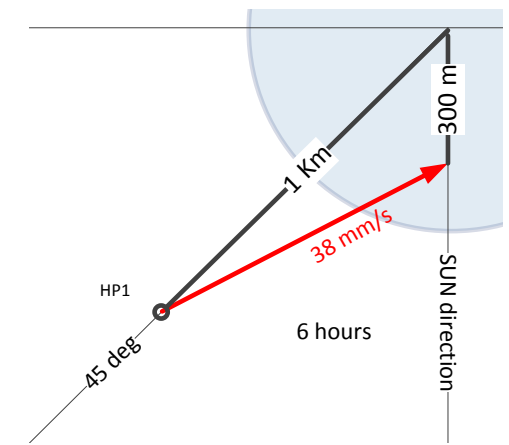

Figure 12. Close Approach Final Segment

\section{Full Operational Phase}

After performing approximately calibration campaigns to test the GNC autonomous system and the laser, the full operative phase starts. The spacecraft maintains its trajectory in the proximity of the asteroid avoiding to being captured by the asteroid. This is performed in order to carry out the asteroid deflection mission, which requires to point the laser beacons towards the asteroid's surface while collecting the solar power. Given these premises, it is convenient to describe the dynamic motion of each spacecraft in the rotating Hill reference frame. In the proximity of the asteroid, the spacecraft is subject to the resulting force due to solar pressure, the gravity of the asteroid, the gravity of the Sun, the centrifugal and Coriolis forces plus other forces induced by the impingement with the plume. Moreover the asteroid is accelerating under the effect of the laser ablation, and, thus, the spacecraft experiences the same acceleration in magnitude but in the opposite direction. 
Following the ellipsoidal asteroid model, we assume that the semi-axis $\mathrm{c} \ell$ is aligned with the $\mathrm{z}$-axis of the asteroid Hill frame A (see Figure 13) and that the asteroid rotates around the z-axis with angular velocity wa 12.

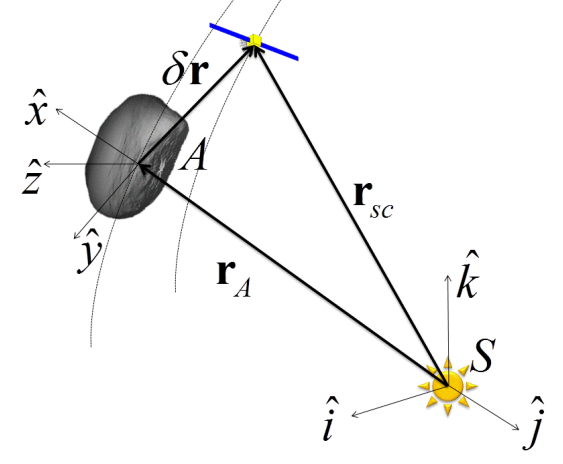

Figure 13. Definition of the reference frames, including the rotating Hill frame A centred on the asteroid.

Assuming the asteroid's shape is an ellipsoid, the gravity field of the asteroid is expressed as the sum of a spherical field plus a second-degree and second-order field ${ }^{9}$

$$
U_{20+22}=\frac{\mu_{A}}{\delta r^{3}}\left(C_{20}\left(1-\frac{3}{2} \cos ^{2} \gamma\right)+3 C_{22} \cos ^{2} \gamma \cos 2 \lambda\right)
$$

where the harmonic coefficients $\mathrm{C} 20$ and $\mathrm{C} 22$ are a function of the semi-axes,

and $\gamma$ is defined as

$$
\begin{aligned}
& C_{20}=-\frac{1}{10}\left(2 c_{l}^{2}-a_{l}^{2}-b_{l}^{2}\right) \\
& C_{22}=\frac{1}{20}\left(a_{l}^{2}-b_{l}^{2}\right)
\end{aligned}
$$

$$
\lambda=\arctan \left(\frac{y}{x}\right)+w_{A}
$$

If one considers a Hill reference frame centred in the barycentre of the asteroid, the motion of the spacecraft in the proximity of the asteroid itself is given by ${ }^{10}$

$$
\ddot{\mathbf{x}}=-\mathbf{a}_{r_{a}}-2 \mathbf{v} \times \dot{\mathbf{x}}-\dot{\mathbf{v}} \times \mathbf{x}-\mathbf{v} \times(\mathbf{v} \times \mathbf{x})-\frac{\mu_{s u n}}{r_{s c}{ }^{3}}\left(\mathbf{x}+\mathbf{x}_{r_{a}}\right)-\frac{\mu_{a}}{\delta r^{3}} \mathbf{x}+\nabla U+\frac{\mathbf{F} s c\left(\mathbf{x}, \mathbf{x}_{r_{a}}\right)}{m_{s c}}
$$

where $\mathbf{a}_{r_{a}}$ is the projection into the local axes of the acceleration the asteroid $\ddot{\mathbf{r}}_{a}$ is subjected to

$$
\ddot{\mathbf{r}}_{a}=-\frac{\mu_{\text {sun }}}{r_{a}^{3}} \mathbf{r}_{a}-\frac{\mu_{S c}}{\delta r^{3}} \mathbf{x}_{a}+\mathbf{a}_{\text {laser }}
$$

The second component on the right side of Eq. (5) represents the tugging effect exerted by the spacecraft on the asteroid, and $\mathbf{a}_{\text {laser }}=f\left(P_{\text {available }}, m_{\text {asteroid }}, A_{\text {spot }}\right.$, composition, kinematics $)$ is the thrust due to the laser ablation process which depends on available power at the laser beam $P_{\text {available }}$, area of the spot on the asteroid $A_{\text {spot }}$, asteroid's mass, composition and spin rate ${ }^{11} ; \mathbf{v}$ is the angular velocity with which the reference frame moves, and it is also affected by the process. In local reference frame its dynamics is given by

$$
\mathbf{x}_{r_{a}} \times\left(\ddot{\mathbf{v}} \times \mathbf{x}_{r_{a}}\right)+2 \dot{\mathbf{x}}_{r_{a}} \times\left(\dot{\mathbf{v}} \times \mathbf{x}_{r_{a}}\right)=\mathbf{x}_{r_{a}} \times \mathbf{a}_{\text {laser-local }}
$$

being $\mathbf{a}_{\text {laser-local }}$ is the projection of $\mathbf{a}_{\text {laser }}$ into the local reference frame. $\mathbf{F}_{s c}\left(\mathbf{x}, \mathbf{x}_{r_{a}}\right)$ includes all the perturbations due to solar radiation pressure, the laser recoil and plume impingement ${ }^{12}$ : 


$$
\begin{aligned}
& F_{\text {Solar }}=C_{R} S_{s r p}\left(\frac{r_{A U}}{r_{s c}}\right)^{2} A_{M} \frac{\mathbf{x}_{a}}{r_{S c}} \\
& F_{\text {recoil }}=\eta_{s y s} S_{s r p}\left(\frac{r_{A U}}{r_{s c}}\right)^{2} A_{M} \frac{\mathbf{x}}{\delta r} \\
& F_{\text {plume }}=\rho_{\text {plume }}(\delta r) \bar{v}_{\text {plume }}^{2}(\delta r) A_{\text {eq }} \frac{\mathbf{x}}{\delta r}
\end{aligned}
$$

where CR is the reflectivity coefficient and SSrp is the solar flux at $1 \mathrm{AU}, \mathrm{AM}$ is the area of the solar arrays, $\mathrm{A}_{\text {eq }}$ is the spacecraft cross section for the plume impingement, $\rho_{\text {plume }}$ and $\bar{v}_{\text {plume }}$ are respectively the plume's density and velocity at the spacecraft (dependant on the distance from the spot ${ }^{11}$ ). The motion of the spacecraft is thus ruled by:

- Laser recoil: Reaction force induced by conservation of momentum upon the projection of laser photons. This force acts to push the spacecraft away from the asteroid.

- Solar radiation pressure: exerted mainly in the $7.4 \mathrm{~m}^{2}$ solar panels, but also partially in the S/C body. The spacecraft is nominally sun pointing, but the nominal value still changes with the distance to the Sun. A part from that, it can be considered a stochastic value where the magnitude changes by $20 \%$ (conservative) with respect to its nominal, where this excursion has a time correlation of 7 days.

- Plume impingement: Caused by the jet of ejecta plume hitting the body and solar panels of the spacecraft. Pushes the spacecraft away from the asteroid. The magnitude depends on the cross section of the exposed surface. If ablating from radial direction ('below'), the ejecta will hit the full back surface of the solar panels and hence be larger than if ablating from trailing position, where it will directly hit the (smaller in area) solar panel shields (the solar panels are perpendicular to the SC-NEO direction).

- Deflection induced: a fictitious force arising from the accelerating local frame. The frame is centred in the asteroid's CoM, which is, through ablation, thrusting with a $10 \mathrm{mN}$ force, thus accelerating at $0.077 \mu \mathrm{m} / \mathrm{s} 2$. The chase caused by this acceleration is equivalent to a force, when seen in a local frame of about $42 \mu \mathrm{N}$.

Table 2 presents the forces characterization at the operating distance of $50 \mathrm{~m}$ at perihelion. At estimation (and simulation) level, these forces are regarded as sum of a bias (fixed value) to a random walk (exponentially correlated random value). The table shows what a typical nominal value should be for the $550 \mathrm{~kg}$ spacecraft and how by much (and at which rate) the value can change from its nominal. The balance is performed for two configurations: trailing the asteroid, where the solar radiation pressure acts at 90 degrees with respect to the others, and from radial direction where it acts against the sum of the others.

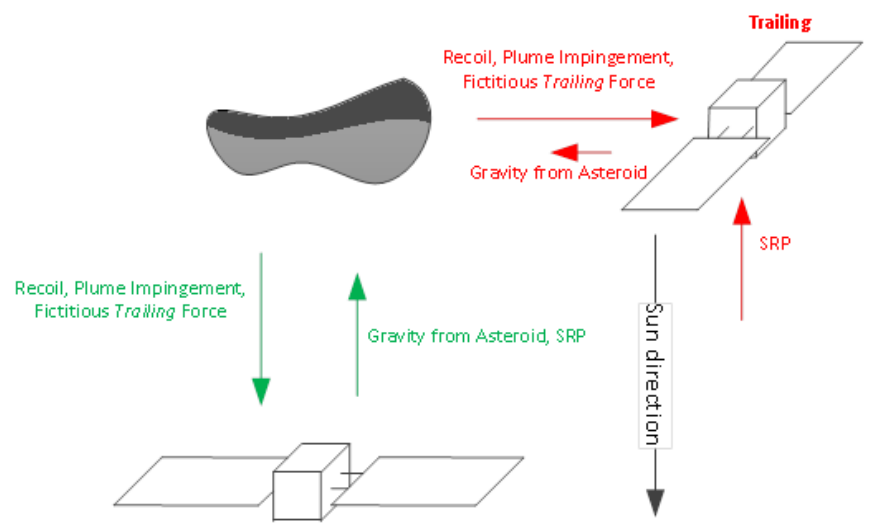

Figure 14. Force directions in trailing and radial configuration

The estimation makes use of this forces makes use of previous heuristic knowledge of the dynamic models of such forces, as well as low pass filters to estimate acceleration / torque components over long time windows. The estimation of these forces allows optimizing actuations to lead the dynamics to limit cycles with maximum excursion with minimum number of actuations. 
The table shows that a significant difference exists in the balance of forces with the radial configuration resulting in having to compensate 1/4th of the force that would have to be compensated in the trailing configuration. Furthermore, the 0 degree illumination phase angle is favourable for navigation, as it minimizes the distance of the $\mathrm{CoB}$ to the actual centroid of the asteroid (and its CoM), as well as its excursion with rotation.

\section{Table 2. Modelling of acting forces at operating distance at perihelion}

\begin{tabular}{|lll|}
\hline & Force $\boldsymbol{\mu N}$ & Variation \\
\hline Solar Radiation Pressure & 38 & $20 \%$ \\
\hline Laser Recoil & 3.3 & $1 \%$ \\
\hline Gravity from Asteroid & 1.7 & $10 \%$ \\
\hline Plume Impingement & $20(6)$ & $20 \%$ \\
\hline Deflection-induced & 42.3 & $20 \%$ \\
\hline Total (trailing) & 62.8 & \\
\hline Total (radial) & 25.5 \\
\hline
\end{tabular}

Beyond the dragging force due to the acceleration of the asteroid and the motion of the frame, the major perturbation is due to the solar radiation acting on the solar mirrors along the $\mathrm{x}$-axis.

\section{E. Measurement model}

On board orbit determination at the asteroid will be performed by combining optical measurements from the camera with the ranging information from the laser range finder ${ }^{13}$. The measurements from the camera are defined on the screen of the camera itself as the coordinates of the asteroid centroid and translated into angular measurements. The definition of the asteroid as seen from the camera a certain number of points are taken the asteroid surface. The position of each point is given in the spacecraft reference frame as:

$$
\mathbf{x}_{\text {Surf-SC }}^{i}=\delta \mathbf{r}_{S C}-\mathbf{x}_{\text {surface }}^{i}
$$

where $\mathbf{x}_{\text {surface }}^{i}$ are the vector position of the points with respect to the centre of the asteroid. Then these points are given in the camera reference frame in the components $\left(x_{c a m}, y_{c a m}, z_{c a m}\right)^{i}$ :

$$
\begin{aligned}
& x_{\text {cam }}^{i}=\mathbf{x}_{\text {Surf }-S C}^{i} \cdot \mathbf{x}_{\text {camera }} \\
& y_{\text {cam }}^{i}=\mathbf{x}_{\text {Surf-SC }}^{i} \cdot \mathbf{y}_{\text {camera }} \\
& z_{\text {cam }}^{i}=\mathbf{x}_{\text {Surf-SC }}^{i} \cdot \mathbf{z}_{\text {camera }}
\end{aligned}
$$

where $\mathbf{x}_{\text {camera }}, \mathbf{y}_{\text {camera }}$ and $\mathbf{z}_{\text {camera }}$ represent the axes of the local camera frame. Being $\mathbf{v}^{i}=\left[\begin{array}{lll}v_{x}^{i} & v_{y}^{i} & v_{z}^{i}\end{array}\right]$ the normalized the local vector, the position of the surface point in terms of pixel can be defined as:

$$
\begin{aligned}
& x_{\text {screen }}^{i}=v_{x}^{i} t_{c} / p_{\text {width }} \\
& y_{\text {screen }}^{i}=v_{y}^{i} t_{c} / p_{\text {width }}
\end{aligned}
$$

where $t_{c}=f / v_{z}^{i}$, $\mathrm{f}$ is the focal length and $p_{\text {width }}$ is the pixel width. The centroid coordinates $\left(x_{c}, y_{c}\right)$ is obtained by the mean position of the all points on the screen of the camera. A representation of this stage of the process is reported in Figure 15 which reports also the position of the centroid with respect to the actual centre. 


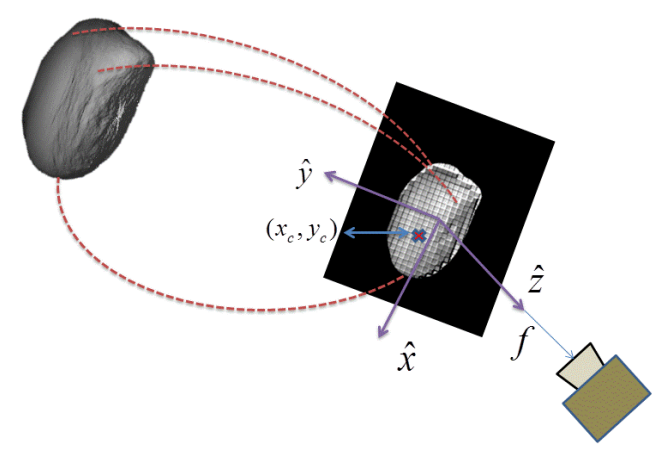

Figure 15. Centroid identification

The local azimuth and elevation angles are obtained as:

$$
\begin{aligned}
& \theta=\tan ^{-1} \frac{x_{c}}{f} \\
& \varphi=\tan ^{-1} \frac{y_{c}}{\sqrt{x_{c}^{2}+f^{2}}}
\end{aligned}
$$

The measurements from the camera results in being affected from both attitude and pixelization errors. The latter is due to the fact that the surface points in terms of pixel are defined as multiple of pixels and could lead to misidentifying the actual pixel position on the camera screen. A minimum of two points on the asteroid surface is necessary to make navigation system observable. When a range measurement is added to a camera image, only one visible surface point is required for the navigation system to be observable. The measurements from the LRF are given by the distance between the spacecraft and the spot the camera is pointing to:

$$
d=\left|\mathbf{x}_{\text {sc }}-\mathbf{x}_{\text {surface }}\right|
$$

where $\mathbf{X}_{\text {surface }}$ is the position of the spot on the asteroid surface.

\section{F. Control law}

The GNC has to assess different definitions of range to cope with mission requirements and its own performances. On one hand, in order to focus the beam the range to surface has to be known in the $0.1 \mathrm{~m}$ range. The ranging sensors also directly measure this quantity, more specifically the range in the direction of the beam's boresight. Combining with LOS measurements, this can directly be converted to altitude, that is, range to surface in the SC-CoM direction. The range to CoM, is the distance between CoM of the spacecraft and of the asteroid. The displacement of the sensor head with respect to the spacecraft's CoM is trivially computed since its attitude and position is well known. It is this quantity that is of interest for translational dynamics. Figure 16 shows how rotation is coupled with the measurements.

The implications for GNC are numerous: on one hand, a measurement is available that (with little filtering - cut-off high frequencies/averaging) can provide $\mathrm{cm}$ accuracy to focusing the beam. On the other hand the translational control cannot react to keep this measurement constant, because that would imply a large amount of useless actuation, back and forth, to keep at the same range to the surface. Furthermore, the states for the implemented navigation filter that describes the translational dynamics regards the relative CoM positions.

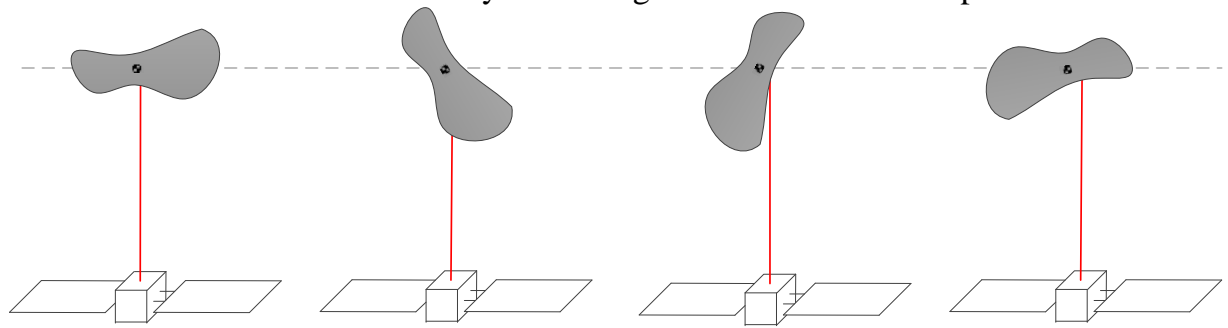

Figure 16. Range to surface change with rotation of the asteroid 
To provide insight to this issue, consider the $2 \mathrm{DoF}$ simplification shown in 16 . The rotation of the asteroid, at maximum, will cause an excursion in range of $\pm 0.5 \mathrm{~m}$, if the CoM distance remains fixed. The ablation laser mechanism has the capability to adjust focus to $\pm 1 \mathrm{~m}$ of the nominal operating distance. Translated to a requirement, this means that the CoM distance needs to be controlled to a box of $\pm 0.5 \mathrm{~m}$. Figure 17 shows how an excursion of $\pm 0.5 \mathrm{~m}$ in CoM plus the rotation lead to an excursion of range to surface of $\pm 1 \mathrm{~m}$.

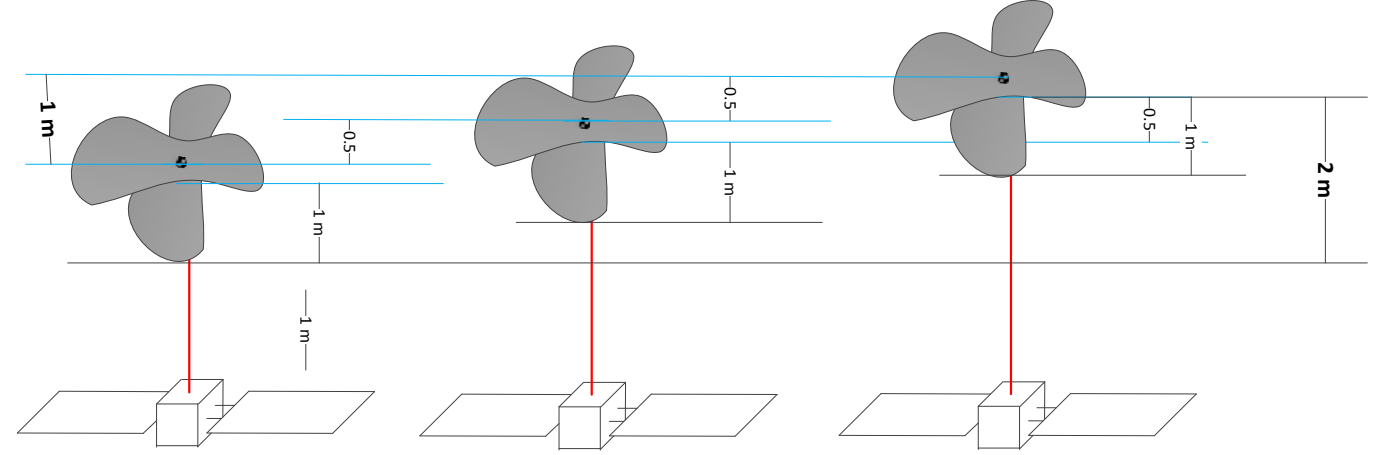

Figure 17. Maximum excursion of range to surface caused by a $1 \mathrm{~m}$ excursion in CoM range and rotation

On setting maximum levels for the activation logic of the proximity controller, a design choice of activating when range to surface measurement exceed $1 \mathrm{~m}$ of its nominal value assures that the logic acts on the quantity to assess. This quantity is the range to surface because that's what concerns the focusing mechanism of the laser.

At each instant of time the autonomous system propagates the estimated state up to the following instant of time. Then the system checks for the inclusion of the spacecraft between the boundaries defined by the control box. The control allocates an impulse bit, keeping into account the estimated acceleration acting on that direction, exploiting the dynamics to reduce the overall number of actuations. The spacecraft tends to follow a parabolic trajectory under the effect of perturbations:

$$
f=\mathbf{d}_{i n}+\left(\mathbf{v}_{\text {in }}+\Delta \mathbf{v}_{c o r r}\right) t+\mathbf{a} \frac{t^{2}}{2}
$$

Where $\mathbf{d}_{\text {in }}$ and $\mathbf{v}_{\text {in }}$ are the initial position and velocity error with respect to the nominal trajectory, $\Delta \mathbf{v}_{c o r r}$ is the corrective impulse bit, while $\mathbf{a}$ is the acceleration. The corrective impulse bit is allocated such that the spacecraft reaches the other side of the control box, with relative velocity equal to 0 .

$$
\begin{aligned}
& f^{\prime}=\mathbf{v}_{i n}^{e s t}+\Delta \mathbf{v}_{\text {corr }}+\mathbf{a}_{\text {est }} t=0 \\
& \mathbf{d}_{f}=\mathbf{d}_{i n}^{\text {est }}+\left(\mathbf{v}_{\text {in }}^{e s t}+\Delta \mathbf{v}_{\text {corr }}\right) t+\mathbf{a}_{\text {est }} \frac{t^{2}}{2}
\end{aligned}
$$

where all the variables have been substituted by their estimated counterpart at the current instant of time. In this way it is assumed a constant value for the perturbations acting on the spacecraft. In order to achieve this limit cycle, an estimate of the relative acceleration is necessary.

A typical trend of the controlled trajectory in the trailing configuration is reported in Figure 18, where one can see the parabolic curves due to both the spacecraft dynamics and the implemented control for a $20 \mathrm{~cm}$ box. 

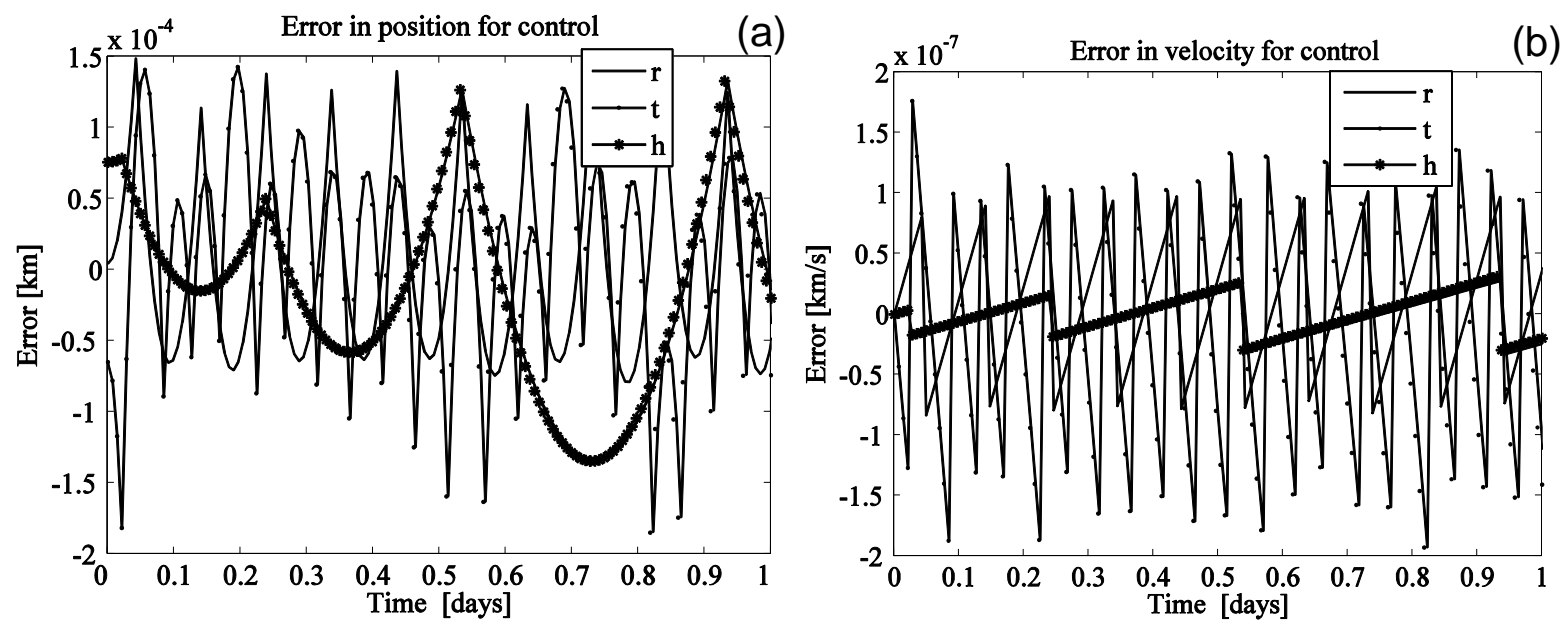

Figure 18. Controlled trajectory for $20 \mathrm{~cm}$ box (a) position, (b) velocity error
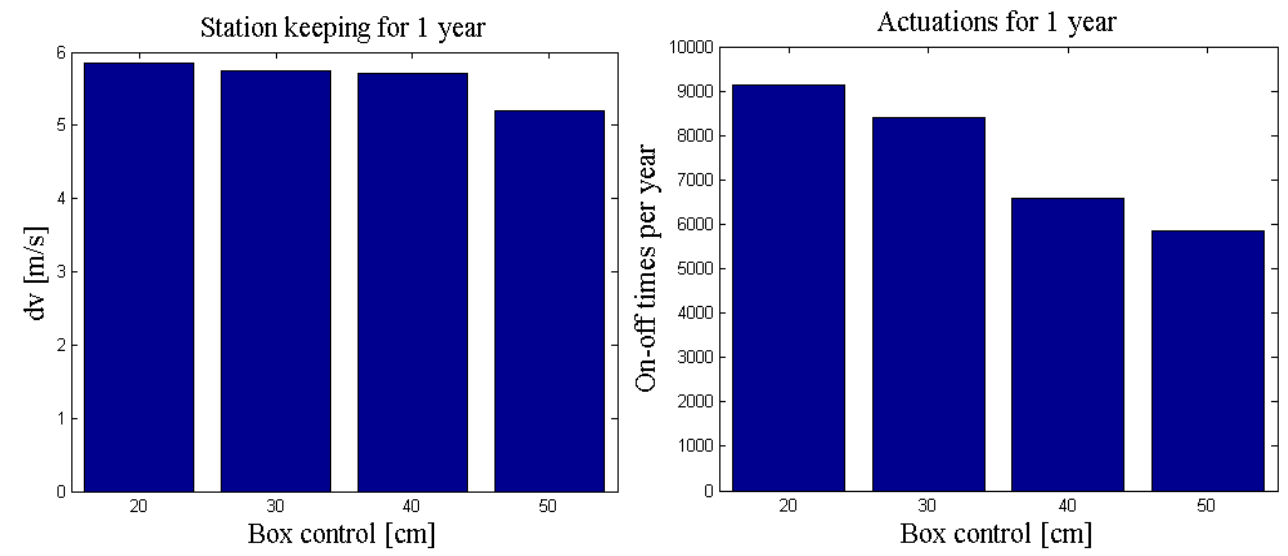

Figure 19. Control in trailing configuration for 1 year ( 365 days) of continuous operations
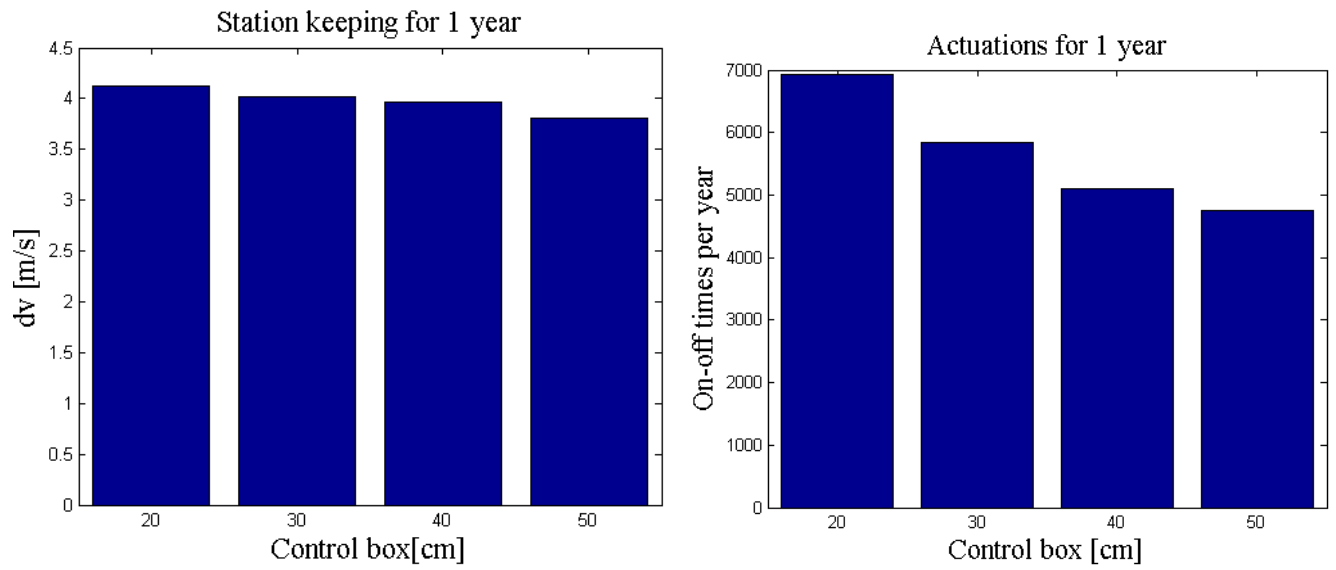

Figure 20. Control in radial configuration (365days) of continuous operations

Figure 19 and Figure 20 reports the necessary $\Delta \mathrm{v}$ required to station-keep the spacecraft at $50 \mathrm{~m}$ in the trailing and radial configuration respectively. It has been assumed that the power available at the laser is constant for the whole period (with no contamination due to the plume). This is a conservative assumptions, because the power at aphelion will be different and the contamination from the plume will reduce the solar arrays' efficiency.

From Figure 20 one can see that the $\Delta \mathrm{v}$ for station keeping decrease by about $2 \mathrm{~m} / \mathrm{s}$, and subsequently the number of actuations is reduced by more than 1,000 . This is due to the fact that, in the radial configuration, the solar radiation 
pressure pushes the spacecraft along the thrust direction, thus partially counteracting the dragging force, the plume impingement and the laser recoil.

In both the configurations, the number of actuations for 1 year operations is limited under the maximum number of actuations for chemical thrusters (circa 40,000 life cycle). The control strategy envisages also to exploits the frequent actuations to desaturate control reaction wheels, thus, keeping the overall number of actuations by RCS thrusters at a minimum.

\section{G. Asteroid's $\Delta \mathbf{v}$ Estimate}

During operations it is vital to measure the effectiveness of the momentum coupling. The on-board GNC system is able to determine the relative acceleration between the spacecraft and the asteroid. The effect of the thrust exerted by the laser ablation produces a dragging force whose effect is coupled with the plume impingement. The impact sensor gives the necessary information on the mass flow and velocity of the ejecta acting on the direction normal to the surface facing the plume. The contribution of the plume is not negligible in particular when the spacecraft flights in the radial configuration, where the surface affected by the plume is about $8 \mathrm{~m}^{2}$. In this case the relative acceleration from the plume accounts for more than $50 \%$ of the acceleration from the dragging force. The premise of this method is that we can rely on precise methods to model the effects from the laser recoil and from the solar radiation pressure. This is the case, because the acceleration from the solar pressure can be filtered and precise estimated will be available during ground station campaign. Moreover during calibration, the laser recoil will be estimated precisely by simply firing the laser in the asteroid direction defocusing the beam, so that it will not produce any laser ablation.

The proposed method consist of augmenting the state variable the on board system needs to estimate during laser operations, by two variables, which represent the dragging force due to the laser ablation and the plume impingement acceleration. In this way the variables, which the filter will need to estimate, $\operatorname{are}\left[x, y, z, v_{x}, v_{y}, v_{z}, a_{\text {laser }}, a_{\text {plume }}\right]$. The approach is the one used to estimate biases, commonly used to estimate solar radiation pressure ${ }^{14}$. The dynamics equations associated to the acceleration from the laser ablation and the plume impingement is thus time independent:

$$
\begin{aligned}
& \dot{a}_{\text {laser }}=0+v_{\text {laser }} \\
& \dot{a}_{\text {plume }}=0+v_{\text {plume }}
\end{aligned}
$$

where $v_{\text {laser }}$ and $v_{\text {plume }}$ are system noises, which means that the dynamics of these variables is driven by the noises. The acceleration from laser is used to update both the spacecraft and the asteroid dynamics in Eqs. (4) and (5). It has been hereafter considered the level of noise on the system equal to the $10 \%$ of the nominal value. Treating these accelerations as biases is a strong assumption because it implies that their dynamics is slowly varying with time. However, by keeping the spacecraft within a small control box, one can maintain the acceleration almost constant, thus limiting the dynamics effects from the laser ablation.

As one can see from Figure 21 (b), when the spacecraft is allowed to move within a bigger control box, the instant estimated acceleration is affected by higher errors, because the thrust drops by circa $30 \%$ in about 30 minutes, a relatively short time span. On the contrary when the spacecraft oscillation are limited as in Figure 21 (a). Similar trend is also shown in where the trailing configuration is reported in Figure 22. The only difference resides in the contribution from the plume, which is approximately a sixth of the one in the radial configuration.
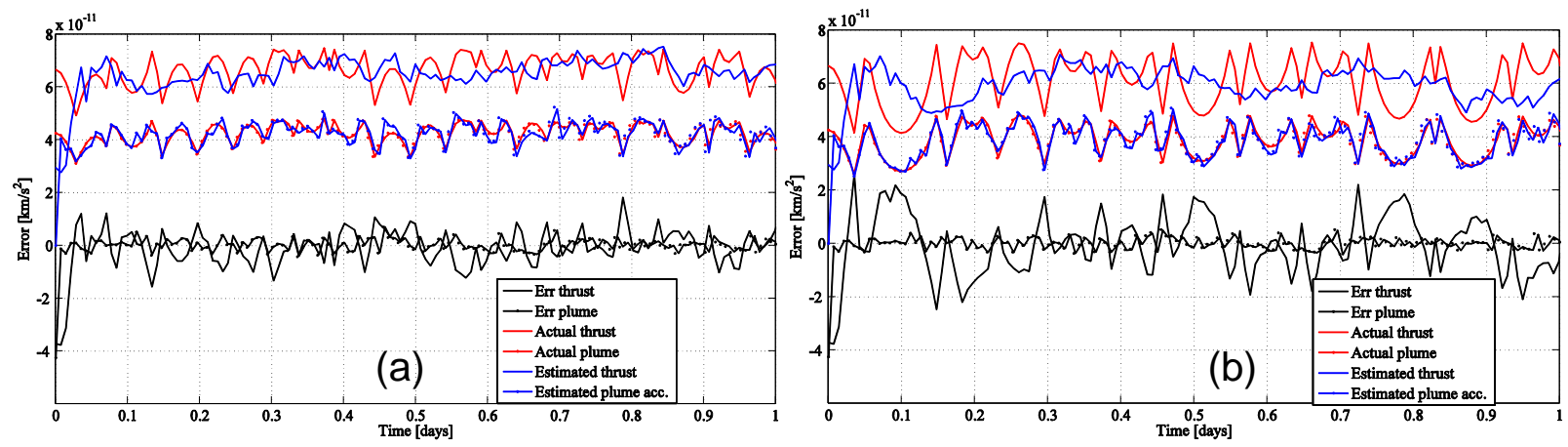

Figure 21. Radial configuration - estimated and actual accelerations with $20 \mathrm{~cm}$ (a) and $50 \mathrm{~cm}$ (b) control box. 
Even though the instant values are different, the integral effects which gives the change in velocity due to the laser are very precise. Table 3 reports the errors in the estimated velocity variation.

Table 3. Velocity variation error form integration over 1 day operations

\begin{tabular}{|lllll|}
\hline & \multicolumn{2}{c}{ Radial } & \multicolumn{2}{c|}{ Trailing } \\
Configuration & \multicolumn{2}{c|}{ configuration } \\
\hline $\begin{array}{l}\text { Control } \\
\text { box }\end{array}$ & $20 \mathrm{~cm}$ & $50 \mathrm{~cm}$ & $20 \mathrm{~cm}$ & $50 \mathrm{~cm}$ \\
\hline $\begin{array}{l}\text { Integral } \\
\text { error }\end{array}$ & $1.6 \%$ & $1.2 \%$ & 0.68 & 0.49 \\
\hline
\end{tabular}

The radial configuration results in being less precise because the contribution from the plume is higher compared to the trailing one. Anyway the estimate is still accurate because lower acceleration for a certain time span will be compensated for and filtered by higher acceleration estimate for a subsequent time span.

The advantage of this method is that there is no assumption on the laser ablation model, which requires the knowledge of a complex dynamics model, involving asteroid kinematics and composition, reaction thermodynamics, a number of variables which cannot be drawn from the embarked payload. Moreover if one integrates the overall contribution of the thrust, one obtains a precise estimate of the velocity imparted to the asteroid. This method is independent from the direction along which the thrust is operating and from the beginning the operations take place.
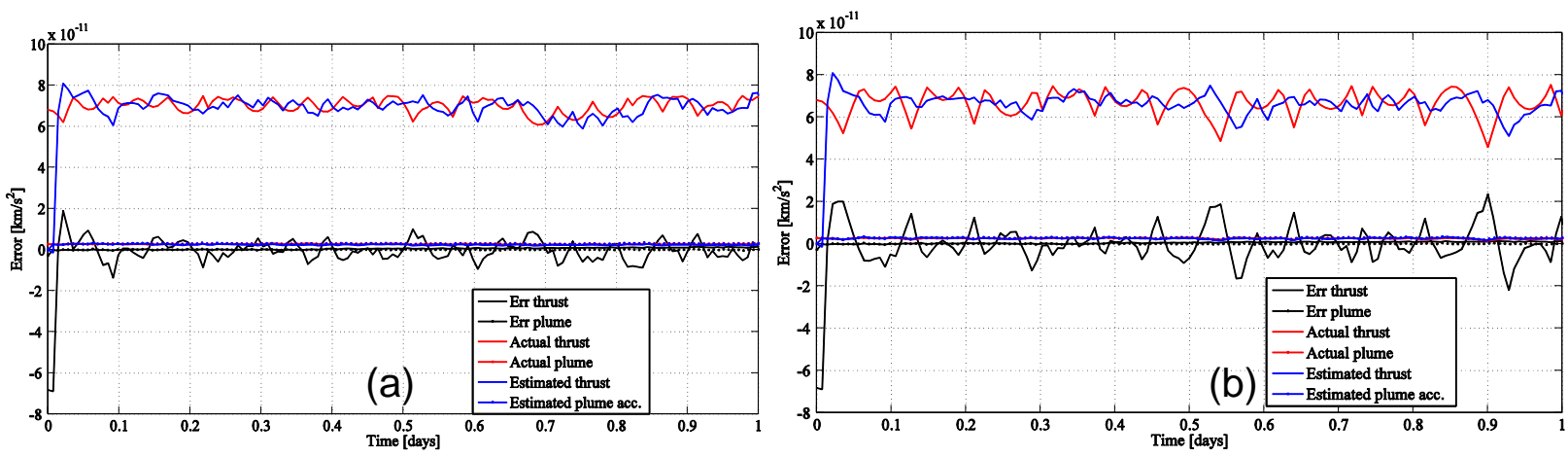

Figure 22. Trailing configuration - estimated and actual accelerations with $20 \mathrm{~cm}$ (a) and $50 \mathrm{~cm}$ (b) control box.

\section{H. Final Remarks}

The paper presented the navigation strategy for the LightTouch2 asteroid deflection mission. Particular attention has been paid to the approach phases, in which the small asteroid, along with its uncertain trajectory represent a challenge for the onboard cameras to detect it. At the same time, the relatively wide area to span before acquiring the faint object on the screen of the camera requires a careful and slow approach. This procedure allows improving the knowledge of the asteroid's ephemeris and spacecraft relative position. Dog-leg manoeuvres have been proposed for this task, basing the whole phase on the expertise of GMV in this field. It has been shown that for asteroid 2006 RH120, a mission launched in 2027 will reach the asteroid two months after the rendez-vous manoeuvre. Once the spacecraft has acquired the target trajectory in the proximity of the asteroid, the ablation process can start. In order to maintain the optimal focussing of the laser, then, the GNC system is required to control the spacecraft within a certain range. This task has been performed by imparting suitable impulsive bits based on the on-board estimate of position, velocity and accelerations. The combination of optical and ranging measurements is employed to achieve this task. In order to on board determine the efficiency of the ablation process, it has been necessary to estimate the imparted acceleration onto the asteroid. This has been achieved by using additional information from the impact sensor to separate the plume impingement contribution from the actual acceleration due to the laser ablation

A simulator accounting for all the forces acting on the spacecraft-asteroid system has been developed. Since the asteroid's spin has noticeable effects on the momentum coupling, future model will foresee the development of an asteroid's attitude model to include also the off-barycentre laser ablation and reduce the spin rate. Optical method will be integrated to obtain an estimate of the asteroid's attitude. 


\section{References}

${ }^{1}$ J.P. Sanchez, C. Colombo, M. Vasile et al. Multicriteria comparison among several mitigation strategies for dangerous near-earth objects. J. Guid. Control Dyn., 32 (2009), pp. 121-141

${ }^{2}$ A. Gibbings, M. Vasile, J.M. Hopkins et al. Potential of laser-induced ablation for future space applications. Space Policy, Volume 28, Issue 3 (2012), Pages 149-153

${ }^{3}$ MarcoPolo-R Payload Definition Document, MarcoPolo-R Study Team, SRE-pA/2011.079, ESA, 2011

${ }^{4}$ Gil-Fernández, J., Cadenas-Gorgojo, R., Prieto-Llanos, T., and Graziano, M., Autonomous GNC Algorithms for Rendezvous Missions to Near-Earth-Objects, AIAA/AAS , Astrodynamics Specialist Conference and Exhibit, Honolulu, HI, 2008.

${ }^{5}$ Persson, S., Jacobsson, B., and Gill, E., PRISMA Demonstration Mission for Advanced Rendezvous and Formation Flying Technologies and Sensors, IAF-05- B5.6.B.07, 56th International Astronautical Congress, Fukuoka, Japan, 2005.

${ }^{6}$ T. V. Peters, et al., Detailed Design of the Proba-3 Formation Flying Guidance, 62nd International Astronautical Congress, IAC-11-C1.7.6, Cape Town, 2011

${ }^{7}$ Guinn, J., Mars Sample Return Navigation: Rendezvous in Mars Orbit, International Symposium for Deep Space Communication; Pasadena, CA; United States, September 21, 1999

${ }^{8}$ Yamanaka, K., Ankersen, F. 2002: New state transition matrix for relative motion on an arbitrary elliptical orbit. J. Guid. Control Dynam. 25(1), 60-66 (2002).

${ }^{9} \mathrm{Hu}$, W. and Scheeres, D. J., July-August 2002. Spacecraft motion about slowly rotating asteroids. Journal of Guidance, Control and Dynamics 25 (4), 765-775.

${ }^{10}$ Schaub, H., Junkins, J. L., 2003. Analytical mechanics of space systems, 1st Edition. AIAA Education Series. AIAA, Virginia, U.S.A.

${ }^{11}$ Kahle, R., Kuhrt, E., Hahn, G., Knollenberg, J., 2006. Physical limits of solar collectors in deflecting Earththreatening asteroids. Aerospace Science and Technology 10, 253-263.

${ }^{12}$ Vasile M., Maddock C., Design of a Formation of Solar Pumped Lasers for Asteroid Deflection, Advances in Space Research, 2012.

${ }^{13}$ Dionne, K., 2009: Improving Autonomous Optical Navigation for Small Body Exploration Using Range Measurements. AIAA 2009-6106. AIAA Guidance, Navigation, and Control Conference, 10 - 13 August 2009, Chicago, Illinois

${ }^{14}$ Maybeck, Peter S. (1979). Stochastic Models, Estimation, and Control. Mathematics in Science and Engineering. 141-1. New York: Academic Press. pp. 423. ISBN 0-12-480701-1. 